\title{
Spinon confinement in a quasi-one-dimensional anisotropic Heisenberg magnet
}

\author{
A. K. Bera, ${ }^{1,2,{ }^{*}}$ B. Lake, ${ }^{1,3, \dagger}$ F. H. L. Essler,${ }^{4, \dagger}$ L. Vanderstraeten, ${ }^{5}$ C. Hubig,${ }^{6}$ U. Schollwöck, ${ }^{6}$ A. T. M. N. Islam, ${ }^{1}$ \\ A. Schneidewind, ${ }^{7}$ and D. L. Quintero-Castro ${ }^{1}$ \\ ${ }^{1}$ Helmholtz-Zentrum Berlin für Materialien und Energie, 14109 Berlin, Germany \\ ${ }^{2}$ Solid State Physics Division, Bhabha Atomic Research Centre, Mumbai 400085, India \\ ${ }^{3}$ Institut für Festkörperphysik, Technische Universität Berlin, 10623 Berlin, Germany \\ ${ }^{4}$ Rudolf Peierls Centre for Theoretical Physics, Oxford University, Oxford OX1 3NP, United Kingdom \\ ${ }^{5}$ Ghent University, Department of Physics and Astronomy, Krijgslaan 281-S9, B-9000 Ghent, Belgium \\ ${ }^{6}$ Arnold Sommerfeld Center for Theoretical Physics, LMU Munich, 80333 München, Germany \\ ${ }^{7}$ Jülich Centre for Neutron Science, Forschungszentrum Jülich GmbH, 85747 Garching, Germany
}

(Received 2 May 2017; revised manuscript received 17 July 2017; published 17 August 2017)

\begin{abstract}
Confinement is a process by which particles with fractional quantum numbers bind together to form quasiparticles with integer quantum numbers. The constituent particles are confined by an attractive interaction whose strength increases with increasing particle separation and, as a consequence, individual particles are not found in isolation. This phenomenon is well known in particle physics where quarks are confined in baryons and mesons. An analogous phenomenon occurs in certain spatially anisotropic magnetic insulators. These can be thought of in terms of weakly coupled chains of spins $S=1 / 2$, and a spin flip thus carries integer spin $S=1$. The collective excitations in these systems, called spinons, turn out to carry fractional spin quantum number $S=1 / 2$. Interestingly, at sufficiently low temperatures the weak coupling between chains can induce an attractive interaction between pairs of spinons that increases with their separation and thus leads to confinement. In this paper, we employ inelastic neutron scattering to investigate the spinon-confinement process in the quasi-one-dimensional, spin-1/2 antiferromagnet with Heisenberg-Ising (XXZ) anisotropy $\operatorname{SrCo}_{2} \mathrm{~V}_{2} \mathrm{O}_{8}$. A wide temperature range both above and below the long-range ordering temperature $T_{N}=5.2 \mathrm{~K}$ is explored. Spinon excitations are observed above $T_{N}$ in quantitative agreement with established theory. Below $T_{N}$ pairs of spinons are confined and two sequences of meson-like bound states with longitudinal and transverse polarizations are observed. Several theoretical approaches are used to explain the data. These are based on a description in terms of a one-dimensional, $S=1 / 2 \mathrm{XXZ}$ antiferromagnetic spin chain, where the interchain couplings are modeled by an effective staggered magnetic mean field. A wide range of exchange anisotropies are investigated and the parameters specific to $\mathrm{SrCo}_{2} \mathrm{~V}_{2} \mathrm{O}_{8}$ are identified. Recently developed theoretical technique based on tangent-space matrix product states gives a very complete description of the data and provides good agreement not only with the energies of the bound modes but also with their intensities. We also successfully explain the effect of temperature on the excitations including the experimentally observed thermally induced resonance between longitudinal modes below $T_{N}$ and the transitions between thermally excited spinon states above $T_{N}$. In summary, our work establishes $\mathrm{SrCo}_{2} \mathrm{~V}_{2} \mathrm{O}_{8}$ as a beautiful paradigm for spinon confinement in a quasi-one-dimensional quantum magnet and provides a comprehensive picture of this process.
\end{abstract}

DOI: 10.1103/PhysRevB.96.054423

\section{INTRODUCTION}

Over the course of the last two decades, quasi-onedimensional (Q1D) quantum magnets have been established as an ideal testing ground for key concepts of quantum many-particle physics such as quantum criticality [1-3], condensation of magnetic excitations [4-8], quantum number fractionalization [9-11], dimensional crossover [12,13], and confinement of elementary particles. Confinement originally arose in the context of high-energy physics as a pivotal property of quarks, but subsequently was realized to emerge quite naturally in one-dimensional quantum many-particle systems and field theories featuring kink or soliton excitations $[14,15]$. The simplest such example involves domain wall ("kink") excitations in Ising-like ferromagnets, and has been explored in exquisite detail in a series of experiments by Coldea and col-

\footnotetext{
*akbera@barc.gov.in

†bella.lake@helmholtz-berlin.de

†Fabian.Essler@physics.ox.ac.uk
}

laborators [16]. Confinement in ladder materials was studied in Ref. [17], while the confinement of spinon excitations has been recently investigated on the Q1D spin-1/2 HeisenbergIsing antiferromagnetic compound $\mathrm{BaCo}_{2} \mathrm{~V}_{2} \mathrm{O}_{8}$ [18]. Here the spinon continuum, characteristic of a 1D spin chain, observed above the three-dimensional ordering temperature $T_{N}$, breaks up into a sequence of gapped, resolution-limited modes in the 3D ordered phase $\left(T<T_{N}\right)$. An interesting difference to the ferromagnetic case (having a single sequence of bound states) is that two sequences of bound states with longitudinal and transverse polarizations, respectively, have been observed.

In the present study we use inelastic neutron scattering to investigate magnetic excitations in the Q1D spin-1/2 XXZ system $\mathrm{SrCo}_{2} \mathrm{~V}_{2} \mathrm{O}_{8}$ as a function of temperature covering both the $1 \mathrm{D}\left(T>T_{N}\right)$ and $3 \mathrm{D}\left(T<T_{N}\right)$ magnetic states. The experimental results are complemented by detailed theoretical considerations that provide a quantitative explanation of the experimental observations.

$\mathrm{SrCo}_{2} \mathrm{~V}_{2} \mathrm{O}_{8}$ crystallizes in the centrosymmetric tetragonal space group $I 4_{1} c d$ (No. 110) with lattice parameters 
(a)

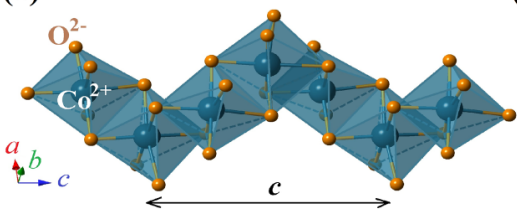

(b)

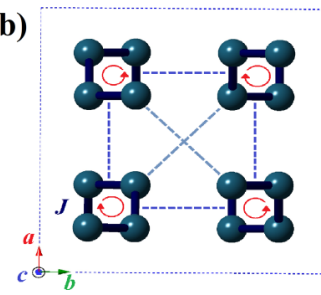

FIG. 1. Crystal structure and interactions of $\mathrm{SrCo}_{2} \mathrm{~V}_{2} \mathrm{O}_{8}$. (a) The screw chain consisting of edge-sharing $\mathrm{CoO}_{6}$ octahedra running along the crystallographic $c$ axis. (b) Projection of the screw chains onto the $a b$ plane. The red arrows show how the chains propagate along the $c$ axis. The intrachain and possible interchain interactions between $\mathrm{Co}^{2+}$ ions are shown by the thick solid and thin dashed lines, respectively.

$a=b=12.2710(1) \AA$ and $c=8.4192(1) \AA$ at room temperature [19]. The magnetic $\mathrm{Co}^{2+}$ ions are situated within $\mathrm{CoO}_{6}$ octahedra which form edge-sharing screw chains along the crystallographic $c$ axis $[19,20]$ [Fig. 1(a)]. There are four screw chains per unit cell which rotate in the $a b$ plane around $(1 / 4,1 / 4),(1 / 4,3 / 4),(3 / 4,1 / 4)$, and (3/4, 3/4) [Fig. 1(b)]. Two diagonal chains rotate clockwise and the other two chains rotate anticlockwise while propagating along the $c$ axis. This results in a complex interaction geometry with many possible superexchange interaction pathways. The strongest interaction is the antiferromagnetic intrachain coupling $J$ between nearest-neighboring $\mathrm{Co}^{2+}$ ions along the chains represented by the solid lines in Fig. 1(b). Examples of the interchain interactions are represented by the dotted lines in Fig. 1(b); many inequivalent interchain interactions are possible due to the screw chain structure. In the isostructural compound $\mathrm{SrNi}_{2} \mathrm{~V}_{2} \mathrm{O}_{8}$, where the screw chains are formed from $\mathrm{Ni}^{2+}$ ions, a total of four interchain interactions were identified [21].

The interchain interactions stabilize long-range collinear antiferromagnetic (AFM) order below $T_{N}=5.2 \mathrm{~K}$ [19] with the spins pointing parallel to the $c$ axis (chain axis). Consecutive spins order antiferromagnetically along the chains while within the $a b$ plane, the spins order ferromagnetically/antiferromagnetically along the $a / b$ axis. The magnetic moment of the $\mathrm{Co}^{2+}$ ions in the distorted octahedral crystal field environment is described well by a highly anisotropic pseudospin, $S=1 / 2$ [22]. The exchange interactions between the pseudospins in $\mathrm{SrCo}_{2} \mathrm{~V}_{2} \mathrm{O}_{8}$ can be approximated by the Hamiltonian [23]

$$
\begin{aligned}
H= & J \sum_{i, j}\left[S_{i, j}^{z} S_{i+1, j}^{z}+\epsilon\left(S_{i, j}^{x} S_{i+1, j}^{x}+S_{i, j}^{y} S_{i+1, j}^{y}\right)\right] \\
& +\sum_{i, j, n, m} J_{n, m}^{i, j}\left[S_{i, j}^{z} S_{n, m}^{z}+\epsilon\left(S_{i, j}^{x} S_{n, m}^{x}+S_{i, j}^{y} S_{n, m}^{y}\right)\right]
\end{aligned}
$$

where the first term is the sum of the Hamiltonians of the individual chains and the second term includes the interchain couplings, and $S_{i, j}^{\alpha}$ is the $\alpha$ component of the $i^{\text {th }}$ spin of the $j^{\text {th }}$ chain. The anisotropy parameter $(\epsilon)$ takes into account the $\mathrm{XXZ}$ anisotropy. For $\mathrm{SrCo}_{2} \mathrm{~V}_{2} \mathrm{O}_{8}$, the intrachain interaction $J$ is antiferromagnetic $(J>0), \epsilon$ can take a value in the range

$0<\epsilon<1$ between the Heisenberg $(\epsilon=1)$ and Ising $(\epsilon=0)$ limits, and the interchain couplings $J_{n, m}^{i, j}$ are weak $\left(J_{n, m}^{i, j} \ll J\right)$.

The present paper is arranged in the following way. Section II gives the experimental details. In Sec. III, the inelastic neutron scattering data for $\mathrm{SrCo}_{2} \mathrm{~V}_{2} \mathrm{O}_{8}$ are presented and analyzed. The magnetic excitations spectrum measured above $T_{N}$ confirms the presence of free spinons, while far below $T_{N}$ bound-spinon modes are revealed. On heating towards $T_{N}$ transitions between the thermally excited bound modes are observed. Theoretical results for the spin-1/2 XXZ AFM chain by several analytical and numerical methods are discussed in Sec. IV. The strong-coupling expansion and field theory approaches are given. The results of numerical methods, i.e., density matrix normalization group (DMRG) and tangentspace matrix product states (MPS) methods, are also shown. A comparison between experimental and theoretical results is given in Sec. V, which shows that a quantitative explanation is provided by the MPS method. The significance of our work is then discussed in Sec. VI.

\section{EXPERIMENTAL METHODS}

Single crystals of $\mathrm{SrCo}_{2} \mathrm{~V}_{2} \mathrm{O}_{8}$ were grown using the floatingzone method [19]. Inelastic neutron scattering (INS) experiments were performed using the cold-neutron tripleaxis spectrometers FLEXX at Helmholtz-Zentrum Berlin, Germany, and PANDA at the Heinz Maier-Leibnitz Zentrum, Garching, Germany. Measurements were performed on a large cylindrical single crystal (weight $\sim 4.5 \mathrm{~g}$, diameter $\sim 4 \mathrm{~mm}$, and length $\sim 40 \mathrm{~mm})$ in the $(h, 0, l)$ reciprocal space plane. The measurements were performed with fixed final wave vectors of $k_{f}=1.3 \AA^{-1}, k_{f}=1.57 \AA^{-1}$, and $k_{f}=1.8 \AA^{-1}$. For these measurements, the sample was mounted on an aluminum sample holder and was cooled in a cryostat. For the FLEXX spectrometer, a double-focusing monochromator and a horizontally focusing analyzer were used. For the PANDA spectrometer, both the monochromator and analyzer were double focusing. Higher order neutrons were filtered out by using a velocity selector on the FLEXX spectrometer and a cooled beryllium filter on the PANDA spectrometer. Measurements took place at various temperatures between $0.8 \mathrm{~K}$ and $6.0 \mathrm{~K}$.

\section{EXPERIMENTAL RESULTS}

\section{A. High-temperature phase $T>T_{N}$}

Since for $\mathrm{SrCo}_{2} \mathrm{~V}_{2} \mathrm{O}_{8} T_{N} /\left(J / k_{B}\right) \ll 1$, we expect there to be a temperature regime $T_{N} \lesssim T \ll\left(J / k_{B}\right)$ in which the physics is essentially one-dimensional (1D) and approximately described by an anisotropic spin-1/2 Heisenberg XXZ chain. Single-crystal inelastic neutron scattering measurements of $\mathrm{SrCo}_{2} \mathrm{~V}_{2} \mathrm{O}_{8}$ at $6 \mathrm{~K}\left(>T_{N}=5.2 \mathrm{~K}\right)$ along the $(0,0, l)$ direction (chain direction) reveal a gapped scattering continuum [Fig. 2(a)]. For such wave vectors the polarization factors are such that only the components of the dynamical structure factor transverse to the direction of magnetic order contribute to the scattering cross section. The gap value of $\simeq 0.95 \mathrm{meV}$ at the $(0,0,2)$ zone center is quite small compared to the bandwidth of the dispersion $(\simeq 14.5 \mathrm{meV}$ ) revealing that the compound 

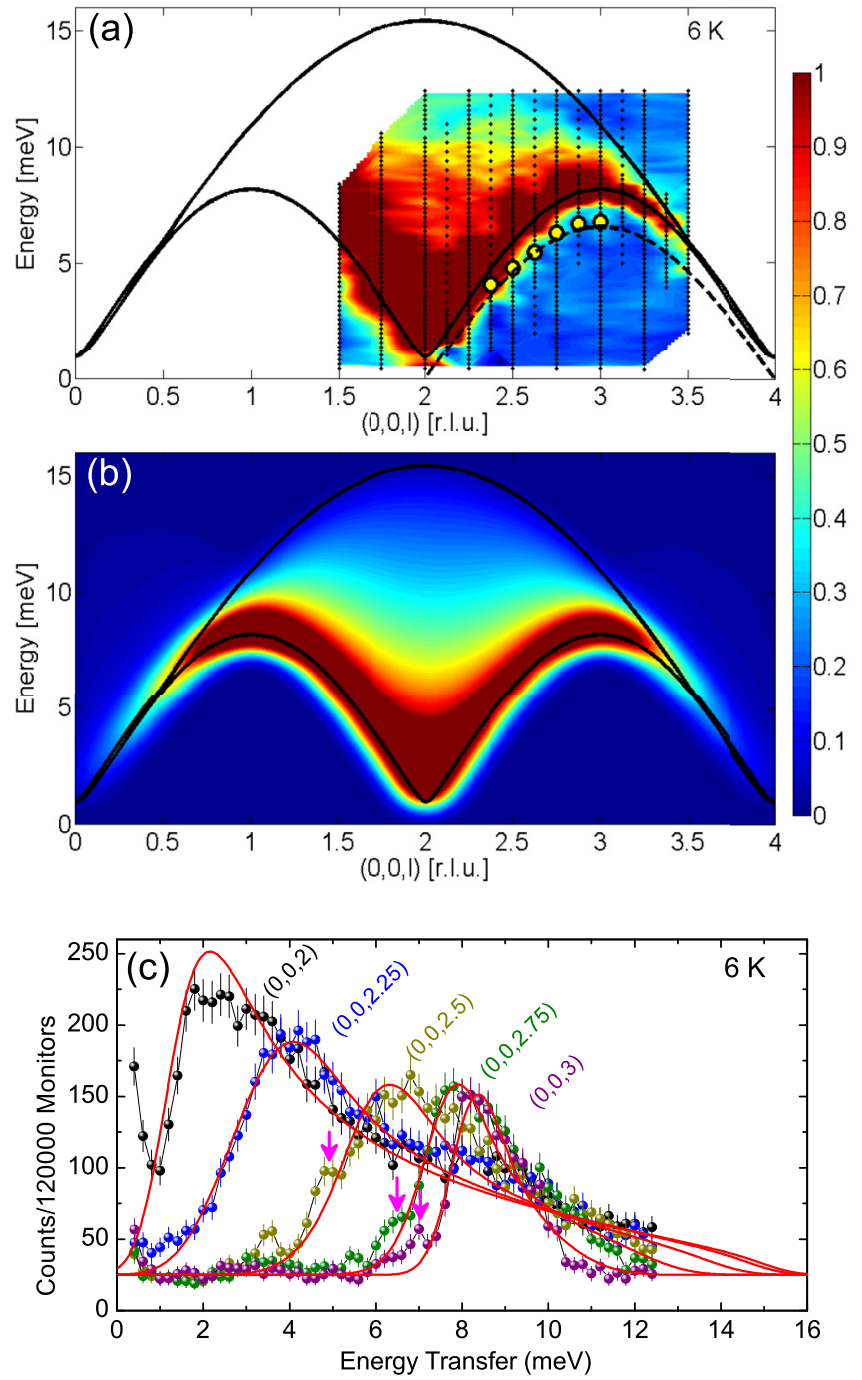

FIG. 2. Inelastic neutron scattering from $\mathrm{SrCO}_{2} \mathrm{~V}_{2} \mathrm{O}_{8}$ measured in the 1D magnetic phase at $6 \mathrm{~K}\left(T>T_{N}\right)$, compared to theory. All the measurements were performed with $k_{f}=1.57 \AA^{-1}$. (a) Magnetic excitation spectrum along the chain axis $(0,0, l)$. The spectrum was obtained by combining several energy scans performed at constant wave vector (the measured data points are represented by the black dots) and the colors indicate the size of the neutron scattering cross section. The solid black lines are the fitted boundaries of the 2spinon continuum of the 1D $S=1 / 2 \mathrm{XXZ}$ AFM calculated from the Bethe ansatz [24] using $J=7.0 \mathrm{meV}$ and $\epsilon=0.56$. The dashes curve is the predicted Villain mode dispersion given by Eq. (6) and the yellow circles give the positions of the weak peaks observed below the continuum in the data. (b) The exact two-spinon contribution to the zero-temperature transverse dynamical structure factor for the 1D $S=1 / 2 \mathrm{XXZ}$ AFM spin chain with $J=7.0 \mathrm{meV}$ and $\epsilon=0.56$ [24] convolved with the instrumental resolution and multiplied by the form factor of $\mathrm{Co}^{2+}$. (c) Energy scans at constant wave vectors of $(0,0,2)$, $(0,0,2.25),(0.0 .2 .5),(0,0,2.75)$, and $(0,0,3)$ measured at $6 \mathrm{~K}$. The arrows point to the observed peaks attributed to the Villain mode. The solid lines through the data are the theoretical intensities of the 1D $S=1 / 2 \mathrm{XXZ}$ AFM spin chain convolved with the instrumental resolution and multiplied by the form factor.

lies intermediate between the Ising $($ gap $\sim J$, bandwidth $\simeq \epsilon J)$ and Heisenberg (gapless, bandwidth $\simeq \pi J$ ) limits.

\section{Spinon continuum at $T>T_{N}$}

The observed spinon continuum at $T=6 \mathrm{~K}\left(\ll J / k_{B}\right)$ is in good agreement with the predictions for the transverse dynamical structure factor of the integrable Heisenberg XXZ chain at zero temperature [24]. The lower boundary of the two-spinon continuum as a function of reduced momentum transfer $0 \leqslant Q<\pi$ along the chain is given by

$$
\omega_{l}(Q)= \begin{cases}\omega_{-}(Q) & \text { for } 0 \leqslant Q<Q_{\kappa}, \\ \frac{2 I}{1+\kappa} \sin (Q) & \text { for } Q_{\kappa}<Q<\pi / 2, \\ \omega_{1 \mathrm{sp}}(Q) & \text { for } \pi / 2<Q<\pi,\end{cases}
$$

and the upper boundary is

$$
\omega_{u}(Q)= \begin{cases}\omega_{1 \mathrm{sp}}(Q) & 0<Q<Q_{\epsilon} \\ \omega_{-}(Q) & Q_{\epsilon}<Q<\pi\end{cases}
$$

Here

$$
\begin{aligned}
\omega_{ \pm}(Q) & =\frac{2 I}{1+\kappa} \sqrt{1+\kappa^{2} \pm 2 \kappa \cos (Q)}, \\
\omega_{1 \mathrm{sp}}(Q) & =I k^{\prime}+I \sqrt{1-\left(1-k^{\prime 2}\right) \cos ^{2}(Q)},
\end{aligned}
$$

where $\kappa=\cos \left(Q_{\kappa}\right)=\frac{1-k^{\prime}}{1+k^{\prime}}, I=J K\left(\sqrt{1-k^{\prime 2}}\right) \sqrt{1-\epsilon^{2}} / \pi$, $K(k)$ is the complete elliptic integral of the first kind, and the parameter $k^{\prime}$ is given by

$$
\frac{K\left(k^{\prime}\right)}{K\left(\sqrt{1-k^{\prime 2}}\right)}=\frac{1}{\pi} \operatorname{arccosh}\left(\epsilon^{-1}\right) .
$$

The specific value $Q_{\epsilon}$ is obtained from the solution of a quartic equation [24].

For $\mathrm{SrCo}_{2} \mathrm{~V}_{2} \mathrm{O}_{8}, Q$ can be written in terms of the crystallographic wave vector transfer $Q^{\prime}$ as $Q=Q_{c}^{\prime} / 4=2 \pi l / 4$, where $Q_{c}^{\prime}$ is the wave vector transfer in terms of the $c$-lattice parameter of $\mathrm{SrCo}_{2} \mathrm{~V}_{2} \mathrm{O}_{8}$. The factor of four arises from the four equivalent $\mathrm{Co}^{2+}$ ions per unit cell along the chain direction ( $c$ axis). Fitting the experimental continuum boundaries of $\mathrm{SrCo}_{2} \mathrm{~V}_{2} \mathrm{O}_{8}$ to the above expressions yields the values of $J \approx$ $7.0 \pm 0.2 \mathrm{meV}$ and $\epsilon \approx 0.56 \pm 0.02$. The fitted continuum boundaries are represented by the solid black lines plotted over the data in Fig. 2(a).

Reference [24] also provides the theoretical expression for the transverse structure factor of the 2-spinon continuum. Using the fitted values of $J$ and $\epsilon$ for $\operatorname{SrCo}_{2} \mathrm{~V}_{2} \mathrm{O}_{8}$, the calculated transverse structure factor is shown in Fig. 2(b) and can be directly compared to the experimental data in Fig. 2(a). Figure 2(c) shows energy scans at several fixed wave vectors from $(0,0,2)$ to $(0,0,3)$ which pass through the lower edge of the continuum of $\mathrm{SrCo}_{2} \mathrm{~V}_{2} \mathrm{O}_{8}$. The lines through the data are the theoretical intensities convolved with the instrumental resolution. Good agreement is achieved between experiment and theory except at $(0,0,2)$ where the effects of interchain coupling and finite temperature, which are not included in the calculation, may alter the spectrum at lowest energies.

\section{Villain mode}

An interesting feature in the dynamical response of spin chains is the existence of a finite-temperature resonance known as a Villain mode [25]. This "mode" was first observed by neutron scattering in Refs. [26,27] and is a fairly general 
feature of spin chain models $[28,29]$. The Villain resonance in the XXZ chain has been investigated theoretically by developing a perturbation theory around the Ising limit [30]. A prediction of this theory is that above a certain temperature, a narrow resonance develops at an energy

$$
\omega_{\mathrm{V}}(Q) \simeq \max _{p}\left|\omega_{1 \mathrm{sp}}(p)-\omega_{1 \mathrm{sp}}(p+Q)\right| .
$$

The resonance corresponds to transitions between thermally occupied states and therefore disappears at zero temperature. In our case, we expect to see a resonance at low temperatures at

$$
\omega_{\mathrm{V}}(Q) \approx 0.94 J \sin (Q),
$$

which follows a similar dispersion to that of the lower boundary of the continuum but is shifted downward from it by an energy similar to the energy gap $\simeq 0.95 \mathrm{meV}$. The predicted Villain mode is indicated in Fig. 2(a) by the dashed black curve. As $T=6 \mathrm{~K}$ which is still quite low compared to the intrachain interaction $\left[T /\left(J / k_{B}\right) \approx 0.07\right]$, we expect the temperature effects on the $T=0$ two-spinon continuum to be weak. Hence, the most noticeable effect of temperature is the emergence of additional peaks associated with the Villain mode just below the two-spinon continuum in the $T=6 \mathrm{~K}$ data. A weak peak is indeed visible in the $(0,0,3)$ data at $\approx 6.8 \mathrm{meV}$ and in the $(0,0,2.75)$ and $(0,0,2.5)$ scans at $\approx 6.3 \mathrm{meV}$ and $\approx 4.8 \mathrm{meV}$, respectively [see Fig. 2(c)]. These peak positions along with those obtained from other energy scans (not shown) are represented by the yellow circles in Fig. 2(a) and follow the predicted Villain mode dispersion given by the dashed black curve.

\section{B. Low-temperature phase $T<T_{N}$}

Below its Néel temperature $T_{N}=5.2 \mathrm{~K}, \mathrm{SrCo}_{2} \mathrm{~V}_{2} \mathrm{O}_{8}$ develops long-range magnetic order where the $\mathrm{Co}^{2+}$ spins order antiferromagnetically along the chains with their moments aligned parallel to the $c$ axis [19]. The dynamical structure factor well inside the ordered phase at $T=1.5 \mathrm{~K}$ along the $(1,0, l)$ direction is shown in Fig. 3. Its gross features including the total bandwidth and the energy gap (minimum) at AFM zone center, i.e., $(1,0,2)$, are similar to those observed above $T_{N}$ [Fig. 2(a)]. An additional weak mode having a similar dispersion with a minimum at the $Q$ point $(1,0,3)$ is also just visible. It is associated with the fact that there are four equivalent screw chains per unit cell each with four $\mathrm{Co}^{2+}$ ions per $c$-lattice parameter. Neglecting interchain interactions this gives rise to a total of four "copies" of the cross section for a single chain, which are shifted with respect to one another by reciprocal lattice units along the chain direction (for details see Ref. [21] on the isostructural compound $\mathrm{SrNi}_{2} \mathrm{~V}_{2} \mathrm{O}_{8}$ ). For uncoupled chains we thus expect the intensity to be of the form

$$
I\left(\mathbf{Q}^{\prime}, \omega\right)=\sum_{l=1}^{4} A_{l}\left(\mathbf{Q}^{\prime}\right) I_{1 \mathrm{D}}\left(4 Q+\frac{2 \pi(l-1)}{c}, \omega\right) .
$$

As a result every reciprocal lattice point is an antiferromagnetic zone center for at least one of these copies, but their overall intensities $A_{l}\left(\mathbf{Q}^{\prime}\right)$ depend on the full momentum transfer $\mathbf{Q}^{\prime}$ and can be very different. For $(1,0, l)$ all four independent contributions are present shifted consecutively by $\Delta Q_{c}^{\prime}=$
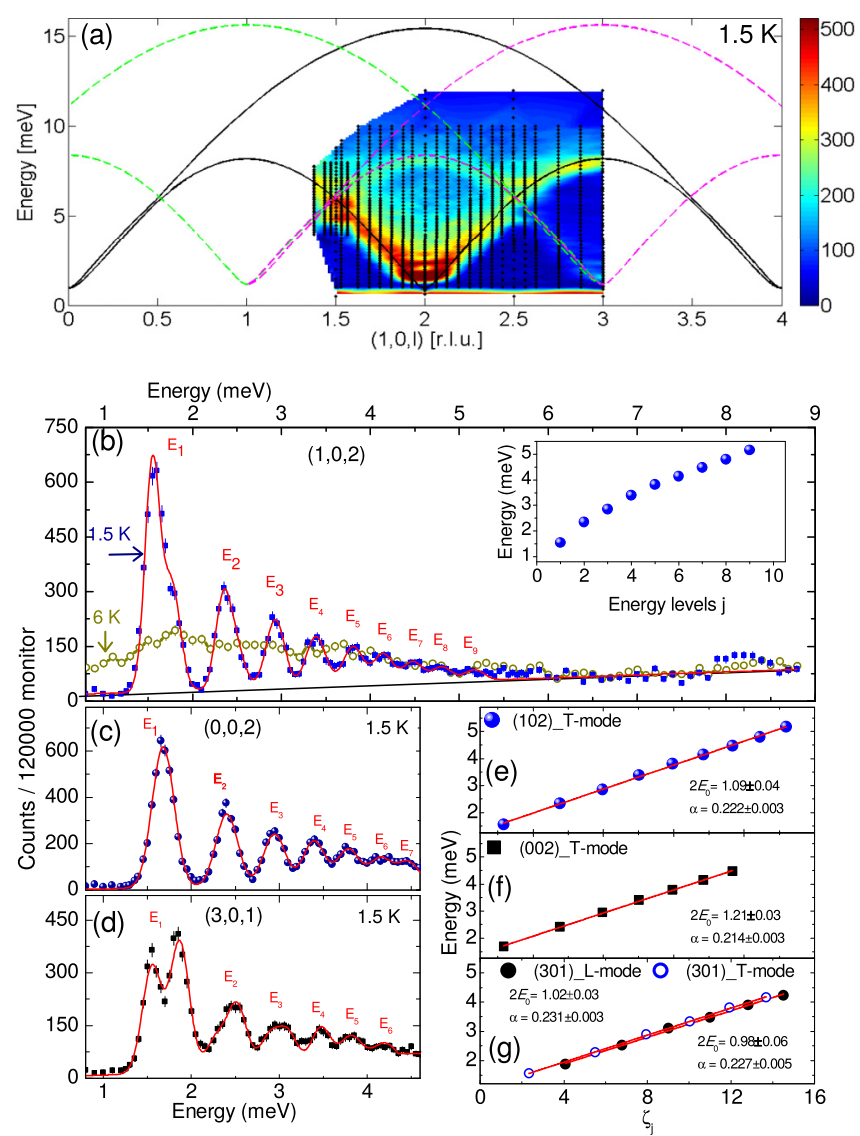

FIG. 3. Neutron scattering data in the ordered phase at $T=$ $1.5 \mathrm{~K}$. (a) Scattering intensity along the chain direction at $(1,0, l)$ measured with $k_{f}=1.8 \AA^{-1}$. The different colored lines delineate the boundaries of the independent and overlapping spectra expected for uncoupled chains. (b) Scans in energy at $(1,0,2)$ for $T=1.5 \mathrm{~K}$ $\left(<T_{N}=5.2 \mathrm{~K}\right)$ and $T=6 \mathrm{~K}\left(>T_{N}\right)$ with $k_{f}=1.57 \AA^{-1}$. The red curve is a fit of Gaussian peaks to the data. The background is shown by the black line. Inset: Energies of the transverse bound-spinon mode excitations as a function of mode number. (c) and (d) Energy scans at constant wave vectors $(0,0,2)$ and $(3,0,1)$ with $k_{f}=1.57 \AA^{-1}$. Only the $\mathrm{T}$ mode is observed at $(0,0,2)$, while both $\mathrm{T}$ mode and $\mathrm{L}$ mode are seen at $(3,0,1)$. The solid red curves are fits of Gaussian peaks to the data. (e)-(g) Fits of the observed bound state energies to a model of two spinons interacting with an attractive interaction increasing linearly with their separation; cf. Sec. III B 1 . The energies of a given series of modes ( $\mathrm{L}$ or $\mathrm{T}$ ) are plotted against the negative zeros of the Airy function $\zeta_{j}$. The solid red lines are the linear fits to the data, demonstrating linear confinement. The fitted values of $E_{0}$ and $\alpha$ are given on the plots.

1 r.l.u. along the chain. Their lower and upper boundaries are indicated by the different colored lines in Fig. 3(a). For $(0,0, l)$ only a single contribution is visible, as observed in Fig. 2(a).

Careful inspection of the cross section at the antiferromagnetic zone center reveals that the continuum observed at $6 \mathrm{~K}$ is transformed into a sequence of discrete, resolution-limited excitations at $1.5 \mathrm{~K}$. As shown in Fig. 3(b) at wave vector transfer $(1,0,2)$ nine peaks, labeled $E_{1}-E_{9}$, are observable in the energy range $\sim 1.5-5.5 \mathrm{meV}$. Since these discrete modes appear below the ordering temperature, they must arise from 

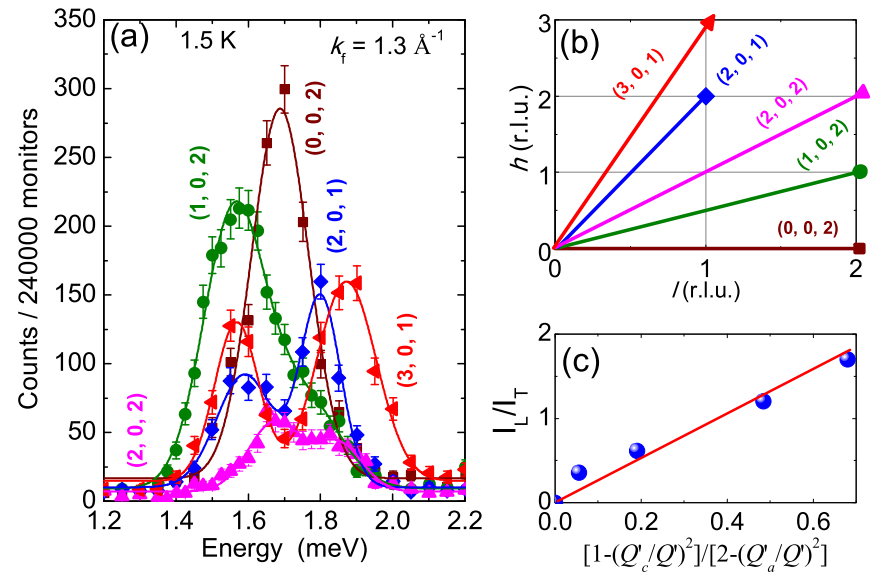

FIG. 4. (a) Constant wave vector scans for several reciprocal lattice points over the lowest pair of bound-spinon modes at $\sim 1.5 \mathrm{meV}$ showing how the $\mathrm{L}$ mode and $\mathrm{T}$ mode intensities vary with wave vector in the $(h, 0, l)$ plane. (b) Directions of the scattering wave vectors in the reciprocal plane for the scans shown in (a). (c) Intensity ratio of the $\mathrm{L}$ mode to the $\mathrm{T}$ mode plotted as a function of $\left[1-\left(Q_{c}^{\prime} / Q^{\prime}\right)^{2}\right] /\left[2-\left(Q_{a}^{\prime} / Q^{\prime}\right)^{2}\right]$. A linear dependence is found (red line) confirming the expected fluctuation directions of the two modes.

the interchain coupling. A detailed examination shows that each of the sharp peaks at $(1,0,2)$ in fact consists of two closely spaced peaks with the higher energy peak being relatively weaker. For the wave vector $(0,0,2)$ a single series of peaks is found [Fig. 3(c)], while at $(3,0,1)$ both series of peaks are visible with similar intensities [Fig. 3(d)].

We have investigated the nature of the two series of peaks in more detail at several AFM zone centers with different wave vector components $Q_{a}^{\prime}$ and $Q_{c}^{\prime}$ (along the $a$ and $c$ axes), respectively. The measurements were performed over the lowest energy peaks around $\sim 1.5 \mathrm{meV}$; see Fig. 4(a). The results indicate that when the wave vector transfer is parallel to the chain direction, e.g., $(0,0,2)$, only one peak is present. If the $a$ component of the wave vector transfer is nonvanishing a second peak appears at higher energy. The relative intensity of the higher energy peak increases with increasing $Q_{a}^{\prime}$.

This intensity dependence provides important information about the nature of the two series of peaks. Neutron scattering is only sensitive to fluctuations perpendicular to the wave vector transfer. Therefore, the longitudinal mode intensity is proportional to $\left[1-\left(Q_{c}^{\prime} / Q^{\prime}\right)^{2}\right]$ while the transverse mode intensity is proportional to $\left[1-\left(Q_{a}^{\prime} /\left|Q^{\prime}\right|\right)^{2}\right]+\left\{1-\left[Q_{b}^{\prime} /\left|Q^{\prime}\right|\right]^{2}\right\}=$ $\left[2-\left(Q_{a}^{\prime} / Q^{\prime}\right)^{2}\right]$ (since there are 2 transverse fluctuations along both the $a$ and $b$ axes and $Q_{b}=0$ ). The higher energy series of modes that is absent for wave vector transfers parallel to the $c$ axis but becomes visible when $Q_{a}^{\prime} \neq 0$ must therefore be due to fluctuations along the $c$ axis. We refer to this series of modes as longitudinal modes ( $\mathrm{L}$ modes) since they are due to fluctuations parallel to the ordered spin direction. In contrast, the available evidence suggests that the lower energy series of modes is associated with fluctuations in the $a b$ plane. We will therefore refer to these excitations as transverse modes (T modes). The intensity ratios of the two modes follow the expected wave vector dependence [see Fig. 4(c)]. The bound modes in $\mathrm{SrCo}_{2} \mathrm{~V}_{2} \mathrm{O}_{8}$ were observed previously using terahertz
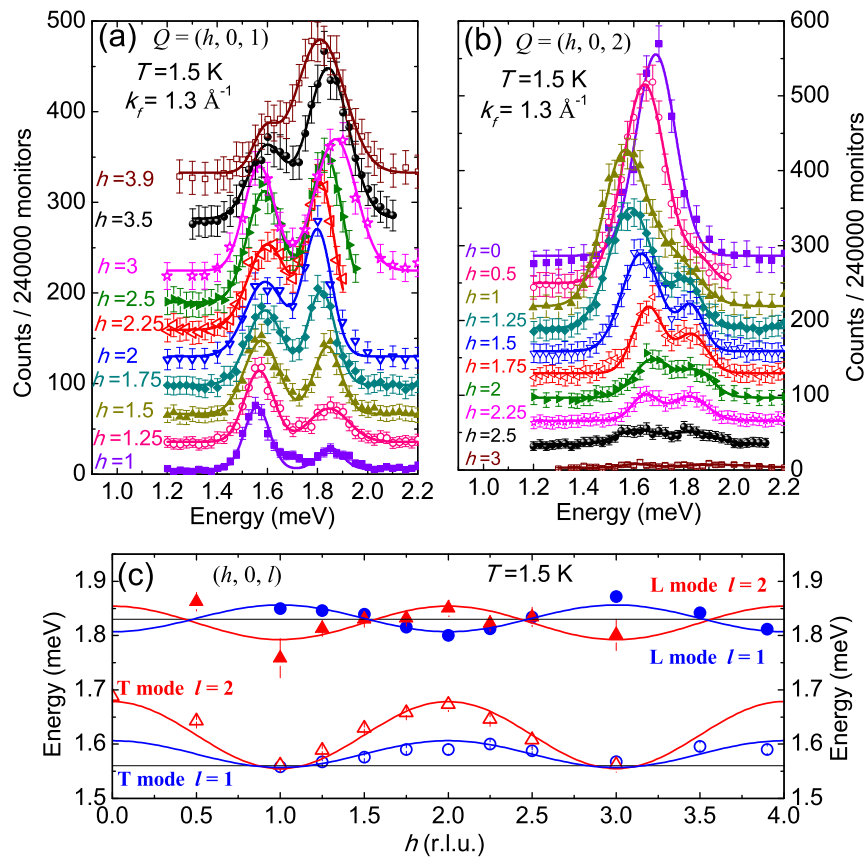

FIG. 5. Constant wave vector scans at $1.5 \mathrm{~K}$ for (a) $(h, 0,1)$ and (b) $(h, 0,2)$. The scans are vertically shifted for clarity. The energy window covers the lowest pair of bound-spinon modes and yields the dispersions perpendicular to the chain axis. The solid lines are fits by sums of two Gaussians and allows the energies of the T and L modes to be extracted. (c) The dispersion relations perpendicular to the chain axis for the T mode and $\mathrm{L}$ mode constructed from the extracted peak positions from (a) and (b). The solid lines are the guides to the eye.

spectroscopy as described in Ref. [31]. This techniques allows the transverse modes to be measured to very high resolution, but the longitudinal modes are not accessible.

It is clear from Fig. 4 that the energies of the modes vary from one AFM zone center to another as a result of the interchain interactions. In order to investigate these interactions, the dispersions of the lowest energy pair of bound modes was measured along $Q_{a}^{\prime}$ by performing a series of energy scans at the constant wave vectors $(h, 0,1)$ and $(h, 0,2)$ for various values of $h$ [Figs. 5(a) and 5(b)]. Both the L mode and $\mathrm{T}$ mode disperse over a narrow bandwidth of $\leqslant 0.15 \mathrm{meV}$; the modes are in-phase for $l=2$ but out-of-phase at $l=1$ [Fig. 5(c)]. The dispersions are complex due to the many possible interchain interactions allowed by the screw-chain crystal structure (Fig. 1) that can reinforce or act against each other depending on the reciprocal lattice points as found for the isostructural compound $\mathrm{SrNi}_{2} \mathrm{~V}_{2} \mathrm{O}_{8}$ [21]. To fully quantify the strengths of the interchain interactions further measurements are required.

\section{Modeling the energies of the observed bound modes}

As we will detail in Sec. IV, the bound states observed at low temperatures can be understood in terms of confinement of spinon pairs. The physical picture is that the interchain coupling induces a linearly confining potential between the elementary spinon excitations of the 1D chains. This was shown by Shiba in Ref. [32] in the large-anisotropy limit $\epsilon \ll 1$ of the model (1). In this limit spinons can be thought of as 
antiferromagnetic domain walls. As we will see in Sec. IV the spinon confinement picture extends all the way up to the Heisenberg limit $\epsilon=1$. This suggests that the bound mode energies $E_{j}$ at the AFM zone center can be approximately extracted from the 1D Schrödinger equation describing the center-of-mass motion of the spinon pairs

$$
-\frac{\hbar^{2}}{\mu} \frac{d^{2} \varphi}{d x^{2}}+\lambda|x| \varphi=\left(E-2 E_{0}\right) \varphi .
$$

Here $\mu$ is the reduced mass, $E_{0}$ is the spinon gap in the absence of the confining potential, $\lambda$ is the molecular field at the $\mathrm{Co}^{2+}$ site produced by the interchain interactions, and the interaction potential between the two spinons is assumed to be a linear function of their separation $x$. The Schrödinger equation (8) has been previously applied successfully to describe aspects of confinement in the transverse field Ising chain [14,15,33-35] and in real materials $[16,18]$. The solutions of Eq. (8) are given by Airy functions [36] and the corresponding bound state energies are

$$
E_{j}=2 E_{0}+\alpha \zeta_{j}, \quad j=1,2,3, \ldots,
$$

where $\alpha=\left[\lambda^{2}\left(\hbar^{2} / \mu\right)\right]^{1 / 3}$ and the $\zeta_{j}$ 's are the negative zeros of the Airy function. We use Eq. (9) as a phenomenological expression for the bound state energies, and fit the two parameters $E_{0}$ and $\alpha$ to our experimental data for the longitudinal and transverse modes separately. This gives excellent agreement with the observed spectra in all cases; see Figs. 3(e)-3(g). The fitted value of $\alpha$ is $\alpha=0.22 \pm 0.1 \mathrm{meV}$ while the spinon gap $0.98<2 E_{0} \mathrm{meV}<1.21$ shows some variation between different AFM zone centers probably due to the interchain coupling.

It should be noted that the bound modes have their minimum at the reciprocal lattice points and disperse along the chain direction as can be observed in Fig. 3(a). The bound mode dispersion in the vicinity of the antiferromagnetic zone center is of the form

$$
\Omega_{j}^{(a)}(Q) \approx E_{j}^{(a)}+\frac{(Q-\pi)^{2}}{2 m_{j}^{(a)}}, \quad a=L, T,
$$

where $Q$ is the reduced wave vector along the chain direction ( $Q=Q_{c}^{\prime} / 4$ ) and $m_{j}$ is the mass of the $j^{\text {th }}$ bound state. In the framework of an effective Schrödinger equation for twospinon excitations one has $m_{j}^{(a)}=E_{j}^{(a)}\left(a_{0} / v\right)^{2}$, where $a_{0}$ is the lattice spacing and $v$ the spinon velocity. We have verified that our data are consistent with such a relationship (Fig. 6).

\section{Temperature effects}

In the simplest model the confining potential for spinons is proportional to the magnitude of the ordered moment and we therefore expect the bound modes to be sensitive to temperature at $T \approx T_{N}$. The temperature dependence of the transverse and longitudinal bound-spinon modes at the reciprocal lattice points $(0,0,2)$ and $(3,0,1)$ is shown in Fig. 7. As temperature approaches $T_{N}$ from below these modes broaden, become weaker, and shift to lower energy. This shift is due to the weakening of the confining molecular field from the neighboring chains as the ordered moment value decreases with increasing temperature.

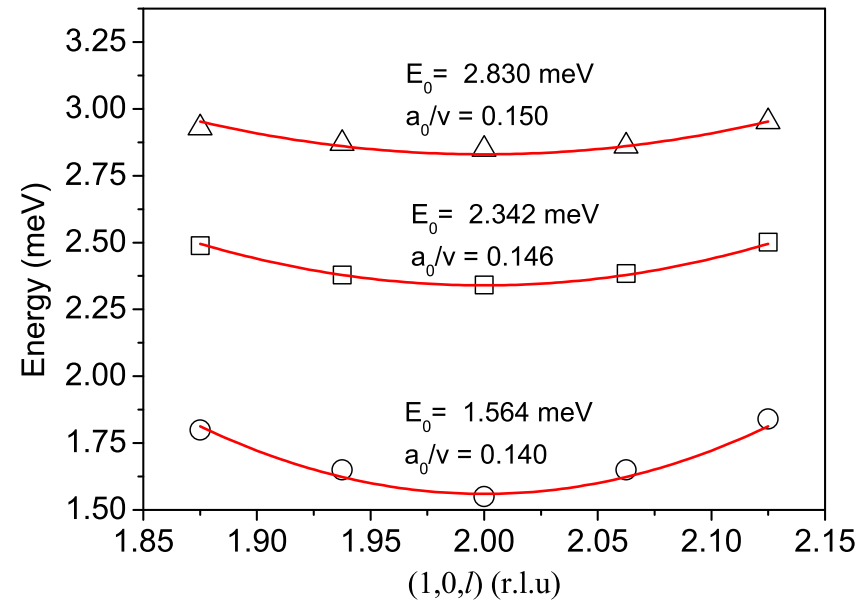

FIG. 6. The dispersions of the three lower energy bound modes close to the zone center $(1,0,2)$. The open symbols are derived from the experimental spectra (Fig. 3) and the red lines are calculated curves as per Eq. (10).

Another feature in the data is a strong broad peak centered around $E=0$ [Fig. 7(b)]. It is visible at $T \sim T_{N}$ but disappears for $T \ll T_{N}$ suggesting that it is due to short-range order between the chains that sharpens into magnetic Bragg peak position well below $T_{N}$. We observe that the peak is present at $(3,0,1)$ but not at $(0,0,2)$. The likely origin of this difference is that at $(0,0,2)$ we only observe transverse correlations, while the peak is related to (emerging) 3D order along the longitudinal direction.

In addition to these changes, a sharp peak appears at $(3,0,1)$ at the energy $E \sim 0.65 \mathrm{meV}$ for $T=4 \mathrm{~K}$ and shifts towards lower energy with increasing temperature. No such temperature-induced peak is observed at $(0,0,2)$, confirming that this feature is associated with the longitudinal structure factor. We attribute this peak to transitions between bound modes. At finite temperatures the lowest energy bound mode will become thermally populated and transitions between it and the higher energy bound modes are possible. Since the peak

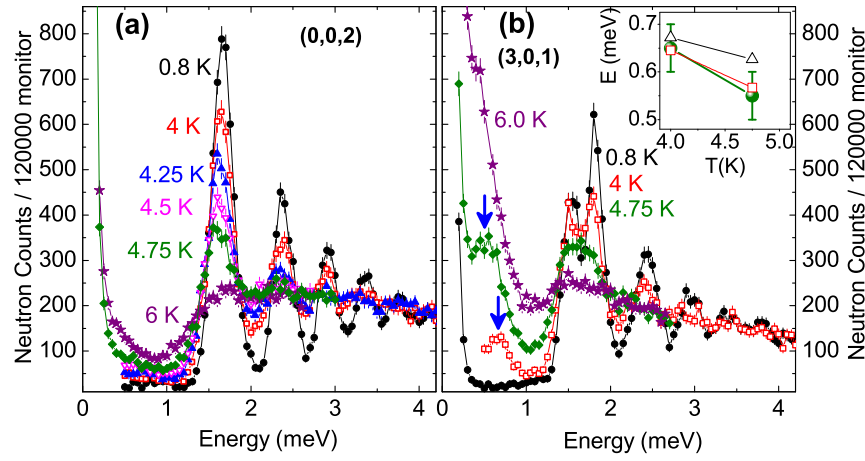

FIG. 7. Energy scans at (a) $(0,0,2)$ and (b) $(3,0,1)$, measured with $k_{f}=1.57 \AA^{-1}$, for several temperatures close to $T_{N}$. Temperatureinduced peaks appear for $(3,0,1)$ and are indicated by arrows. The inset shows the energy of the temperature-induced peak as a function of temperature (green spheres). The energy differences between the first two longitudinal and two transverse bound states are shown by red squares and black triangles, respectively. 
is observed in the longitudinal structure factor it arises from a transition between longitudinal bound modes or between transverse bound modes but not from a longitudinal to a transverse bound mode or vice versa. The distinction should be drawn between this feature which is a transition between thermally excited bound-spinon modes observed close to but below $T_{N}$ and the Villain mode which arises from transitions between thermally excited single spinon states which are observed above $T_{N}$.

If we denote the dispersion relation of the $j^{\text {th }}$ transverse/longitudinal bound state by $\Omega_{j}^{(a)}(Q)(a=T, L)$, transitions between them occur at energies

$$
\Omega_{j, k}^{(a \rightarrow b)}(Q-P)=\left|\Omega_{j}^{(a)}(Q)-\Omega_{k}^{(b)}(P)\right|, \quad a, b=T, L .
$$

In the low-temperature ordered phase $\Omega_{j}^{(a)}(Q)$ will be $\pi$ periodic functions, so that the momentum transfer of the transition (11) will be $Q-P \bmod \pi$. If we take $Q=P=\pi$ there will be transitions with momentum transfer zero and $\pi$. Setting aside the issue of which transitions will give nonvanishing contributions to the dynamical structure factor (which is $2 \pi$-periodic), we have checked whether the energy differences between bound modes at $(3,0,1)$ are reflected in the energy of the temperature-induced peaks. The inset of Fig. 7(b) shows the temperature-dependent energy of the thermally excited peak (green circles) which is in good agreement with the energy difference between the first and second longitudinal bound modes as a function of temperature (red squares). This confirms that the origin of the temperature-induced peak is the transition between thermally populated lowest energy longitudinal bound modes. The above interpretation of temperature effects is supported by the theoretical analysis summarized in Sec. IV D 2.

\section{THEORY}

As we have seen above, at $T>T_{N}$ the neutron scattering intensity is well described by a model of uncoupled spin$1 / 2$ Heisenberg XXZ chains. At low temperatures interchain coupling effects are obviously important. In the following we constrain our analysis to a simple mean-field treatment of these interactions [32,37-40]:

$$
\begin{gathered}
\sum_{i, j, n, m} J_{n, m}^{i, j}\left[S_{i, j}^{z} S_{n, m}^{z}+\epsilon\left(S_{i, j}^{x} S_{n, m}^{x}+S_{i, j}^{y} S_{n, m}^{y}\right)\right] \\
\rightarrow \sum_{i, j, n, m} J_{n, m}^{i, j}\left[\left(S_{i, j}^{z}\right\rangle S_{n, m}^{z}+S_{i, j}^{z}\left\langle S_{n, m}^{z}\right\rangle\right] .
\end{gathered}
$$

Using the fact that there is Néel order at low temperatures this leads to a description in terms of decoupled chains in a self-consistent staggered magnetic field:

$$
H_{\mathrm{MF}}=J \sum_{j=1}^{L} S_{j}^{z} S_{j+1}^{z}+\epsilon\left(S_{j}^{x} S_{j+1}^{x}+S_{j}^{y} S_{j+1}^{y}\right)-h \sum_{j=1}^{L}(-1)^{j} S_{j}^{z} .
$$

The effective staggered field $h$ is a function of $J_{n, m}^{i, j}$ and temperature. We note that the Hamiltonian (13) has a U(1) symmetry of rotations around the $z$ axis:

$$
\left[H_{\mathrm{MF}}, S^{z}\right]=0 \text {. }
$$

In the following we analyze the dynamical structure factor in the model (13) by several different methods in various parameter regimes.

\section{A. Strong-coupling expansion}

A fairly comprehensive qualitative picture of the physical properties of the model (13) can be obtained by considering the strong-anisotropy limit $\epsilon \ll 1$. This limit is amenable to an analysis by the method of Ishimura and Shiba [41] and has been previously considered by Shiba [32]. As Ref. [32] only considered the transverse component of the dynamical structure factor, we now give a self-contained discussion of this approach and then discuss the resulting picture for dynamical correlations. We find it convenient to map (13) to a ferromagnet by rotating the spin quantization axis on all odd sites around the $x$ axis by 180 degrees:

$$
S_{2 j+1}^{a}=-\frac{1}{2} \tau_{2 j+1}^{a}, \quad a=y, z, \quad S_{2 j+1}^{x}=\frac{1}{2} \tau_{2 j+1}^{x} .
$$

Here $\tau_{j}^{\alpha}$ are Pauli matrices. In terms of the new spins we have $H_{\mathrm{MF}}=H_{0}+H^{\prime}$ with

$$
\begin{aligned}
& H_{0}=-\frac{J}{4} \sum_{j} \tau_{j}^{z} \tau_{j+1}^{z}, \\
& H^{\prime}=\frac{J \epsilon}{2} \sum_{j} \tau_{j}^{+} \tau_{j+1}^{+}+\tau_{j}^{-} \tau_{j+1}^{-}-\frac{h}{2} \sum_{j} \tau_{j}^{z},
\end{aligned}
$$

where $\tau^{ \pm}=\frac{\tau^{x} \pm i \tau^{y}}{2}$. The U(1) symmetry (14) gives rise to the commutation relations

$$
\left[H_{\mathrm{MF}}, \sum_{j}(-1)^{j} \tau_{j}^{z}\right]=0 .
$$

The zero-temperature dynamical susceptibilities are given by

$$
\begin{aligned}
\chi^{\alpha \beta}(\omega, Q)= & -\frac{i}{L} \int_{0}^{\infty} d t \sum_{j, l} e^{i \omega t+i Q(j-l)}\left\langle\left[\tau_{l}^{\alpha}(t), \tau_{j}^{\beta}\right]\right\rangle \\
= & -\left\langle\mathrm{GS}\left|\tau_{Q}^{\beta} \frac{1}{\omega+H_{\mathrm{MF}}-E_{0}+i \eta} \tau_{-Q}^{\alpha}\right| \mathrm{GS}\right\rangle \\
& +\left\langle\mathrm{GS}\left|\tau_{-Q}^{\alpha} \frac{1}{\omega-H_{\mathrm{MF}}+E_{0}+i \eta} \tau_{Q}^{\beta}\right| \mathrm{GS}\right\rangle,
\end{aligned}
$$

where $\eta>0$ is infinitesimal, $E_{0}$ is the ground state energy, and $\tau_{Q}^{\alpha}=\frac{1}{\sqrt{L}} \sum_{j} e^{i Q j} \tau_{j}^{\alpha}$. The dynamical structure factor of the antiferromagnetic spin chain (13) of interest is

$$
\begin{aligned}
& S_{\mathrm{AFM}}^{x x}(\omega, Q)=S_{\mathrm{AFM}}^{y y}(\omega, Q)=-\frac{1}{4 \pi} \operatorname{Im} \chi^{x x}(\omega, Q), \\
& S_{\mathrm{AFM}}^{z z}(\omega, Q)=-\frac{1}{4 \pi} \operatorname{Im} \chi^{z z}(\omega, Q+\pi) .
\end{aligned}
$$

We will analyze (18) by carrying out a strong-coupling expansion in the limit $\epsilon, h \ll J$ [41]. Our starting point is the Ising part $H_{0}$ of the mean-field Hamiltonian. The ground states of $H_{0}$ are simply the saturated ferromagnetic states $|\uparrow\rangle$ and $|\downarrow\rangle$, respectively. Their energies are $E_{0}^{(0)}=-\frac{J L}{4}$. Spontaneous symmetry breaking selects, e.g., $|\uparrow\rangle$. The lowlying excitations are then two domain wall states of the 


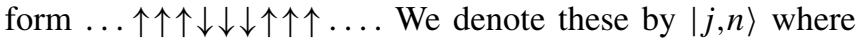
$j$ is the position of the first down spin and $j+n-1$ the position of the last down spin. The energies of these states are $E_{2}^{(0)}=-\frac{J L}{4}+J$. A convenient orthonormal basis of two domain wall states with momentum $Q$ is obtained by taking appropriate linear combinations

$$
|Q, n\rangle=\frac{1}{\sqrt{L}} \sum_{j} e^{i Q j}|j, n\rangle .
$$

The matrix elements of the Hamiltonian in these states are

$$
\begin{aligned}
\left\langle Q, m\left|H_{M F}\right| Q, n\right\rangle & =\frac{J \epsilon}{2}\left(1+e^{2 i Q}\right)\left[\delta_{m, n+2}+e^{-2 i Q} \delta_{m, n-2}\right] \\
& +\left[\left(-\frac{J}{4}-\frac{h}{2}\right) L+J+h n\right] \delta_{n, m} .
\end{aligned}
$$

Importantly, the only nonzero matrix elements in $H_{\mathrm{MF}}$ occur between domain wall states with both even or both odd lengths. This is a consequence of the U(1) symmetry (17) and expresses the fact that acting with $H_{\mathrm{MF}}$ does not change the staggered magnetization (or equivalently the magnetization in the original $S_{j}^{\alpha}$ spin variables). Given (21) it is a straightforward matter to numerically compute the Green's functions:

$$
G(j, k)=\left\langle Q, j\left|\left(\omega-H_{\mathrm{MF}}+E_{0}+i \eta\right)^{-1}\right| Q, k\right\rangle .
$$

\section{Ground state in perturbation theory}

The first order correction to the ground state is obtained by standard perturbation theory

$$
|\mathrm{GS}\rangle \simeq|\uparrow\rangle-\frac{\epsilon \sqrt{L}}{2}|Q=0,2\rangle .
$$

This gives the following matrix elements of spin operators:

$$
\begin{aligned}
\left\langle Q, n\left|\tau_{Q}^{x}\right| \mathrm{GS}\right\rangle & =[1-\epsilon \cos (Q)] \delta_{n, 1}-\frac{\epsilon\left(1+e^{2 i}\right)}{2} \delta_{n, 3} \\
\left\langle Q, n\left|\tau_{Q}^{z}\right| \mathrm{GS}\right\rangle & =\epsilon\left(1+e^{i Q}\right) \delta_{n, 2} \delta_{Q, 0} \\
\left\langle\mathrm{GS}\left|\tau_{Q}^{z}\right| \mathrm{GS}\right\rangle & =\sqrt{L} \delta_{Q, 0} .
\end{aligned}
$$

\section{Dynamical structure factor}

Substituting (24) into (18) then leads to the following approximate expression for the dynamical susceptibilities at $\omega>0$ :

$$
\begin{aligned}
\chi^{x x}(\omega, Q) \simeq & G(1,1)[1-\epsilon \cos (Q)]^{2}+G(3,3)|\epsilon \cos (Q)|^{2} \\
& -G(1,3)[1-\epsilon \cos (Q)] \frac{\epsilon\left(1+e^{2 i Q}\right)}{2} \\
& -G(3,1)[1-\epsilon \cos (Q)] \frac{\epsilon\left(1+e^{-2 i}\right)}{2} \\
\chi^{y y}(\omega, Q)= & \chi^{x x}(\omega, Q+\pi), \\
\chi^{z z}(\omega, Q) \simeq & G(2,2)|2 \epsilon \cos (Q / 2)|^{2} .
\end{aligned}
$$

We note that these are consistent with the U(1) symmetry of rotations around the $z$ axis for the antiferromagnetic model (13). It is now straightforward to compute the dynamical structure factor (DSF) (19) numerically. Results for momentum transfer $\pi$ are shown in Fig. 8 . (a)

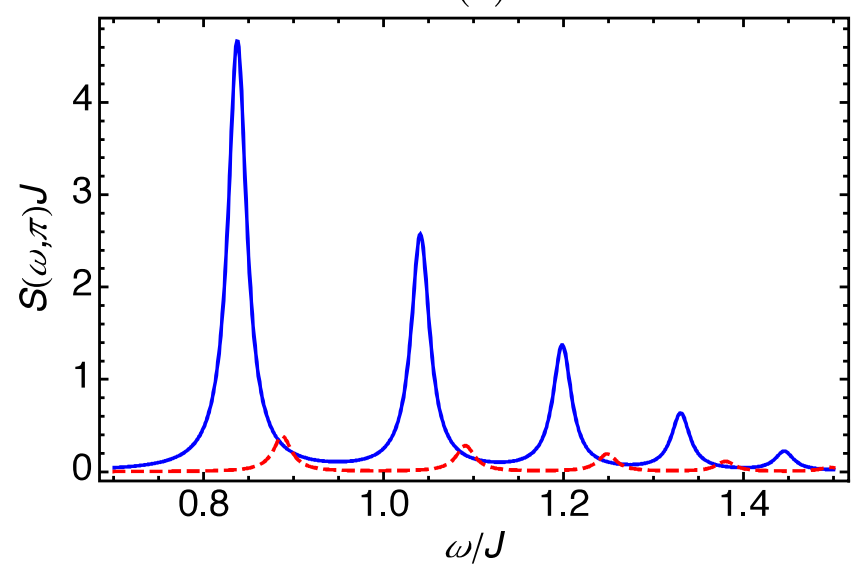

(b)

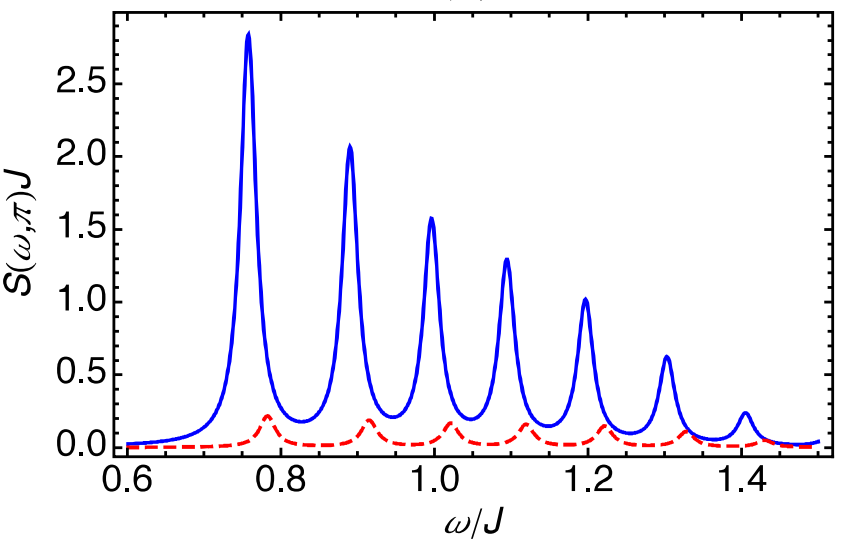

FIG. 8. Transverse DSF $S_{\mathrm{AFM}}^{x x}(\omega, \pi)$ (solid blue line) and longitudinal DSF $S_{\mathrm{AFM}}^{z z}(\omega, \pi)$ (dashed red line) for (a) $\epsilon=0.2$ and $h=0.05 J$, and (b) $\epsilon=0.2$ and $h=0.025 J$. The broadening has been chosen as $\eta=J / 80$ in order to make the $\delta$-function peaks visible.

We see that the transverse $\operatorname{DSF}\left[S_{\mathrm{AFM}}^{x x}(\omega, \pi)\right]$ only "couples" to half the bound states, while the longitudinal DSF $\left[S_{\mathrm{AFM}}^{z z}(\omega, \pi)\right]$ is sensitive to the other half. This is in perfect correspondence with the experimental observations. The selection rule that gives rise to this behavior is related to the conserved $S^{z}$ quantum number (14). It is clear from (21) that the Hamiltonian in the two domain wall sector is block diagonal in a basis of domain wall states of odd/even length. In terms of the original spins even/odd length domain walls correspond to even/odd values of the conserved $S^{z}$ quantum number (assuming the lattice length to be divisible by 4 ). This implies that there is one sequence of bound states with $S^{z}=0$, and a second with $S^{z}= \pm 1$. The first is visible in the longitudinal structure factor $S_{\mathrm{AFM}}^{z z}$, while the second contributes only to $S_{\mathrm{AFM}}^{x x}$. In the strong-anisotropy limit we therefore have the simple cartoon picture for the physical nature of the bound states shown in Fig. 9.

\section{Gap as a function of field}

The energy value of the first peak at $Q=\pi, E_{1}^{(T)}$, gives the excitation gap. Based on the relation of our problem to a 
Ground state

$\hat{\uparrow}-\downarrow-\hat{\jmath}-\downarrow-\hat{\uparrow}-\downarrow-\hat{\uparrow}-\downarrow-\hat{\uparrow}-\downarrow-\hat{\uparrow}-\downarrow-\hat{\uparrow}-\downarrow-\hat{\uparrow}$

1st transverse bound mode T1

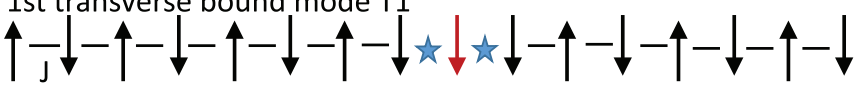

1st Longitudinal bound mode L1

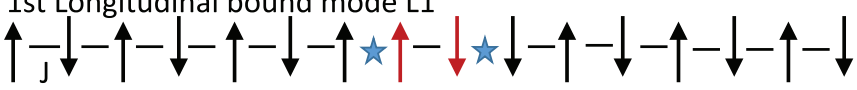

2nd transverse bound mode $\mathrm{T} 2$

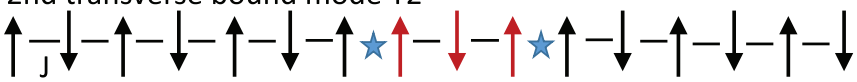

2nd Longitudinal bound mode $\mathrm{L} 2$

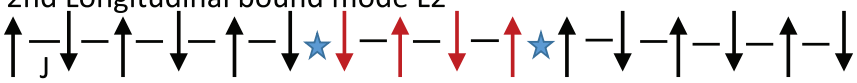

3rd transverse bound mode T3

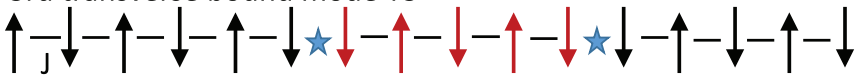

FIG. 9. Cartoon picture of the spin excitations for $\epsilon \ll 1$. (a) Lowest energy configuration showing antiferromagnetic spin alignment. (b) Flipping one spin (red arrow) creates two ferromagnetic domain walls (blue stars). At $T>T_{N}$ these "spinons" can propagate independently and are observed in the INS data as a continuum. At $T<T_{N}$ the domain walls are confined by the molecular field due to the ordering of the neighboring chains: the energy cost increases linearly with the number of reversed spins (red arrows), which leads to the hierarchy of bound modes observed in the INS data. Domain walls separated by an even (odd) number of flipped spins have $S^{z}=0$ $\left(S^{z}=1\right)$ and are observed in the longitudinal (transverse) structure factor.

Schrödinger equation with linear potential we expect

$$
E_{1}^{(T)}=a_{0}+a_{1} h^{\frac{2}{3}}
$$

In Fig. 10 we show the results obtained in our strong-coupling expansion and a fit to (26), which is seen to give a very good account of the data (see Fig. 11).

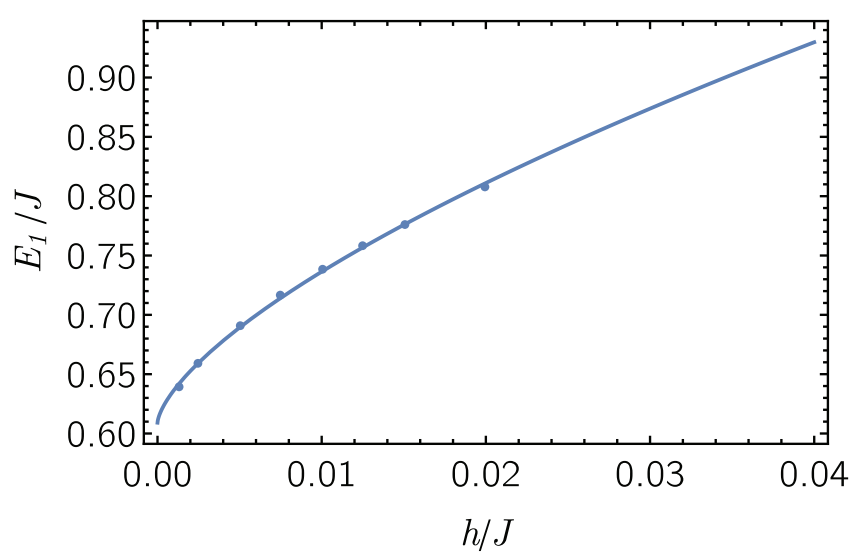

FIG. 10. Gap between the ground state and the lowest excitation at $Q=\pi$ as a function of the staggered field $h$ for $\epsilon=0.2$. Dots are results of the strong-coupling expansion while the solid line is a fit to Eq. (26).

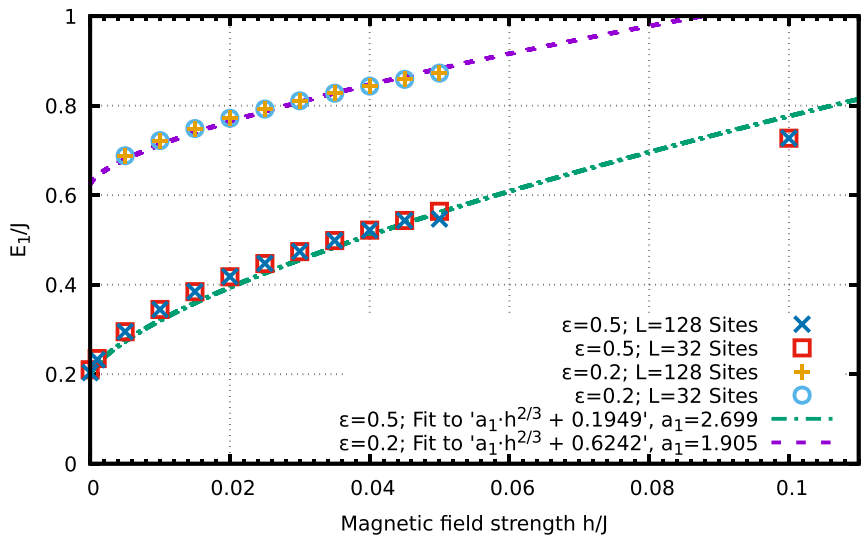

FIG. 11. Energy gap $E_{1}$ of the first bound state as a function of the magnetic field for $\epsilon=0.5$ and $\epsilon=0.2$. The lines are fits to the functional form (26), where the zero-field gap has been computed from the exact solution and $a_{1}=2.699$ and $a_{1}=0.6242$ for $\epsilon=0.5$ and $\epsilon=0.2$, respectively. The finite-size effects between system sizes $L=32$ and $L=128$ are already too small to resolve graphically in most cases.

\section{B. Field theory in the vicinity of the isotropic point $\epsilon=1$}

The physical picture obtained in the large-anisotropy limit $\epsilon \ll 1$ remains valid in the entire regime $0<\epsilon<1$. To see this we consider the limit of weak anisotropy $\epsilon \approx 1$, where the mean-field Hamiltonian (13) can be written in the form

$$
H_{\text {latt }}=J \sum_{j} \boldsymbol{S}_{j} \cdot \boldsymbol{S}_{j+1}+\delta \sum_{j} S_{j}^{z} S_{j+1}^{z}-h \sum_{j}(-1)^{j} S_{j}^{z},
$$

where $\delta \approx J(1-\epsilon)$. In the parameter regime $h \ll \delta \ll J$ this model can be bosonized following, e.g., $[42,43]$, which leads to a two-frequency sine-Gordon model

$$
\begin{aligned}
\mathcal{H}= & \frac{v}{2} \int d x\left[\left(\partial_{x} \Phi\right)^{2}+\left(\partial_{x} \Theta\right)^{2}\right] \\
& +\int d x[\lambda \cos \sqrt{8 \pi} \Phi(x)+\mu \sin \sqrt{2 \pi} \Phi(x)],
\end{aligned}
$$

where $\lambda \propto \delta$ and $\mu \propto h$. The model (28) in the relevant parameter regime has been studied previously by a number of authors $[34,44,45]$. A fruitful line of attack is to start with $\mathcal{H}$ for $\mu=0$, and consider the $\mu$ term as a perturbation. The minima of the potential for $\mu=0$ and $\lambda>0$ occur at

$$
\Phi(x)=(2 n+1) \sqrt{\frac{\pi}{8}}, \quad n \in \mathbb{Z} .
$$

The solutions to the classical equations of motion are solitons and antisolitons. Solitons interpolate between neighboring vacua, e.g.,

$$
\Phi(x \rightarrow \pm \infty)=\mp \sqrt{\frac{\pi}{8}},
$$

while antisolitons have the opposite asymptotics,

$$
\Phi(x \rightarrow \pm \infty)= \pm \sqrt{\frac{\pi}{8}} .
$$


At the quantum level solitons and antisolitons turn into elementary excitations of the sine-Gordon model.

\section{Soliton-antisoliton states}

Following [44,45] we start with the soliton-antisoliton sector. We take the positions of the soliton and antisoliton to be $x_{1}$ and $x_{2}$, respectively, and denote the classical energy for $\mu=0$ by $2 \Delta_{s}$. When $\mu>0$, soliton-antisoliton states acquire an extra contribution to the energy

$$
2 \mu\left|x_{1}-x_{2}\right|
$$

In a nonrelativistic approximation we then obtain a singleparticle Schrödinger equation for the relative motion $(x=$ $\left.x_{2}-x_{1}\right)$ with Hamiltonian

$$
H_{\mathrm{rel}}=-\frac{1}{M_{s}} \frac{d^{2}}{d x^{2}}+2 \mu|x|
$$

Here $M_{s}=\Delta_{s} / v^{2}$ and the reduced mass is $\frac{M_{s} M_{\bar{s}}}{M_{\S}+M_{\bar{s}}}=\frac{M_{s}}{2}$. This Schrödinger equation can be solved exactly in terms of Airy functions [36], and the corresponding eigenstates describe the confinement on solitons and antisolitons. The bound state energies follow from the boundary conditions imposed on the wave function. If we require the wave function to be antisymmetric and therefore vanish at zero, we obtain

$$
E_{n}^{(L)}=2 M_{s}+\left(\frac{4 \mu^{2}}{M_{s}}\right)^{\frac{1}{3}} \xi_{n}, \quad \operatorname{Ai}\left(-\xi_{n}\right)=0
$$

Symmetric wave functions would instead lead to a spectrum of the form

$$
E_{n}^{(L)}=2 M_{s}+\left(\frac{4 \mu^{2}}{M_{s}}\right)^{\frac{1}{3}} \zeta_{n}, \quad \mathrm{Ai}^{\prime}\left(-\zeta_{n}\right)=0 .
$$

As soliton-antisoliton states have the same $S^{z}$ value as the ground state, transitions between the ground state and the bound states (34) will contribute to the longitudinal structure factor.

\section{Soliton-soliton states}

The considerations for two-soliton states are analogous. Classically the parameter $\mu$ characterizing the confining potential (32) is the same as in the soliton-antisoliton sector, but we do not expect this to be true at the quantum level. We account for this by a different strength $\bar{\mu}$ of the potential, which then gives a sequence of energies

$$
E_{n}^{(T)}=2 M_{s}+\left(\frac{4 \bar{\mu}^{2}}{M_{s}}\right)^{\frac{1}{3}} \xi_{n}, \quad \operatorname{Ai}\left(-\xi_{n}\right)=0
$$

Here we have taken the wave function to be antisymmetric because the zero-momentum limit of the soliton-soliton scattering matrix is -1 . As soliton-soliton states have $S^{z}=1$, transitions between the ground state and the bound states (36) will contribute to the transverse structure factor.

\section{Dynamical structure factor at $Q \approx \pi / a_{0}$}

In the field theory limit the staggered magnetizations are given by

$$
\boldsymbol{n}(t, x)=\left(\begin{array}{c}
\cos [\sqrt{2 \pi} \Theta(t, x)] \\
\sin [\sqrt{2 \pi} \Theta(t, x)] \\
\sin [\sqrt{2 \pi} \Phi(t, x)]
\end{array}\right) .
$$

Close to the antiferromagnetic wave number $\pi / a_{0}$ (where $a_{0}$ is the lattice spacing) the components of the DSF are thus given by

$$
S^{\alpha \alpha}\left(\omega, \frac{\pi}{a_{0}}+q\right) \propto \int_{-\infty}^{\infty} d t d x e^{i \omega t-i q x}\left\langle n^{\alpha}(t, x) n^{\alpha}(0,0)\right\rangle .
$$

In the longitudinal structure factor we therefore see confined soliton-antisoliton states, while the transverse components are sensitive to confined soliton-soliton and antisoliton-antisoliton states. Following Appendix B of [45] we can derive expressions for the bound state contributions to the various correlators in leading order in perturbation theory in $\mu$ in the limit of very weak confinement:

$$
\begin{aligned}
& \left\langle n^{z}(\tau, x) n^{z}(0,0)\right\rangle \\
& \quad \propto \sum_{n=1}^{N_{b}}\left(E_{n+1}^{(L)}-E_{n}^{(L)}\right) \rho\left(E_{n}^{(L)}\right) K_{0}\left(E_{n}^{(L)}\left|x^{2}+v^{2} \tau^{2}\right|\right),
\end{aligned}
$$

where

$$
\rho(E)=\frac{2 f\left[\operatorname{arccosh}\left(E / 2 M_{s}\right)\right]}{\pi^{2} \sqrt{E^{2}-4 M_{s}^{2}}} .
$$

The function $f(\theta)$ is related to a particular two-particle form factor

$$
\begin{aligned}
f(\theta)= & \left.\left|\left\langle 0\left|n^{z}(0,0)\right| \theta_{1}, \theta_{2}\right\rangle_{s \bar{s}}\right|^{2}\right|_{\theta_{1}-\theta_{2}=\theta} \\
= & C \frac{\sinh ^{2}(\theta)}{\sinh ^{2}(3 \theta / 2)} \\
& \times \exp \left[-2 \int_{0}^{\infty} \frac{d x}{x} \frac{\left[\cosh x \cos \left(\frac{x \theta}{\pi}\right)-1\right] \cosh \left(\frac{x}{6}\right)}{\cosh \left(\frac{x}{2}\right) \sinh x}\right] .
\end{aligned}
$$

A similar analysis can in principle also be carried out on the level of the spin chain itself. The strengths of the confining potentials for spinon-antispinon and spinon-spinon two-particle states should be extracted from the known 4-particle form factor.

\section{DMRG results}

While the strong-coupling expansion and field theory analysis provide a good qualitative picture of the dynamics, they do not apply quantitatively to the experimentally relevant regime $\epsilon \approx 0.56$. In order to overcome this shortcoming we have carried out numerical DMRG [46-48] calculations with the SYTEN tensor toolkit, based on the Hamiltonian (13). We first determine the gap of the lowest bound state in the $S^{z}=1$ sector as the difference between the lowest energies of the $S^{z}=0$ and $S^{z}=1$ sectors. This computation is extremely 
stable, even near criticality at small values of the field $h$ and with periodic boundary conditions. Finite-size effects can also be entirely removed by choosing sufficiently large system sizes. We are not able to determine the gaps of higher bound states in this way as this would require working at a fixed momentum. Figure 11 gives the resulting values for the gap and a fit to the small- $h$ prediction (26). We see that already for system sizes $L=32$ the gap value is essentially converged and is in excellent agreement with the theoretical predictions for the scaling (26).

Second, we can calculate the ground state on a long chain, apply an excitation in the middle of the chain, and then use matrix product state-based Krylov time evolution [49] with matrix re-orthogonalization to evaluate the dynamical structure factors in the time-space domain. The Fourier transformation into momentum space is unproblematic. However, we are only able to evolve up to a time $t_{\max } J \approx 80$ for $\epsilon=0.2$. This limitation is a consequence of the entanglement growth during time evolution and the subsequent exponential increase in computational effort. This limit necessitates an artificial damping factor $\exp \left(-\eta \frac{t}{t_{\max }}\right)$ to be introduced during the Fourier transform into frequency space. For $\epsilon=0.2, \eta \approx 1$ suffices and it is already possible to distinguish the physical peaks from the spectral leakage introduced by the transformation. For $\epsilon=0.5$, only slightly shorter time scales $t_{\max } J \approx 50$ are achievable. Sufficient damping to remove spectral leakage then also removes the signal. To circumvent this problem, we use numerical extrapolation prior to the Fourier transformation to extend the data in time to very large $t_{\max }^{\prime} J \approx 1000$. We can then introduce a very small damping $\eta=1 / 700$ during the Fourier transformation and still remove all spectral leakage.

In Fig. 12 we show results for the dynamical structure factor at momentum $Q=\pi$ on a system of $L=128$ sites with $h=0.05 \mathrm{~J}$ and two values of the anisotropy $\epsilon$. In both cases we find two sequences of bound states associated with the transverse and longitudinal correlations, respectively. The position of the first peak in the transverse sector is in good agreement with Fig. 11.

\section{Tangent-space MPS methods}

As we have explained in the previous section, targeting higher bound states variationally requires the ability to work within a fixed momentum sector. This is made possible by using tangent-space methods [50] for matrix product states (MPS) that work directly in the thermodynamic limit. In particular, starting from a translationally invariant MPS ground state on an infinite chain, we can apply the MPS quasiparticle ansatz [51] to target the elementary excitations corresponding to isolated branches in the spectrum. This ansatz can be read as the MPS version of the Feynman-Bijl ansatz and single-mode approximation, but improves on these approaches in using the virtual degrees of freedom of the MPS to build an excitation on top of the ground state. As the ansatz explicitly contains a fixed momentum, it allows us to systematically capture the wave functions of all quasiparticle excitations-the ones that contribute a $\delta$ peak in the DSF-within a certain momentum sector. In order to capture continuous bands in the spectrum, multiparticle excitations should be considered [52,53]. Since the quasiparticle ansatz yields accurate variational expressions (a)

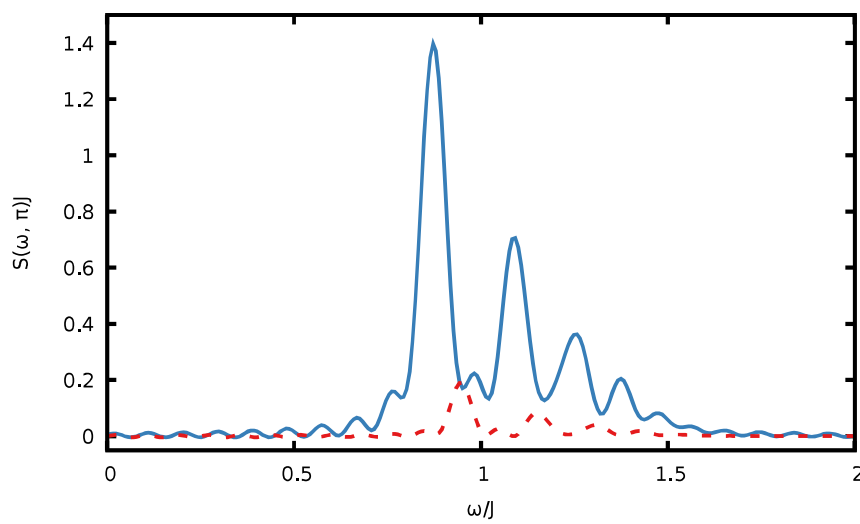

(b)

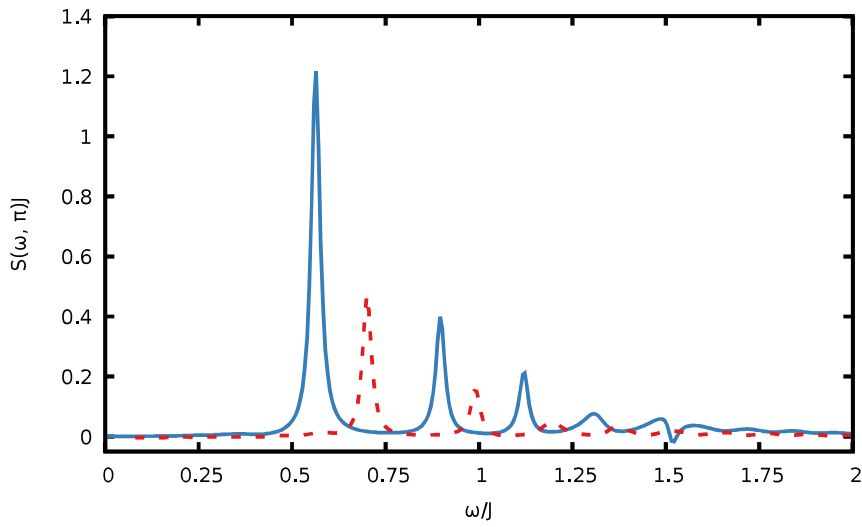

FIG. 12. Transverse (solid blue line) and longitudinal (dashed red line) dynamical structure factors calculated using MPS-Krylov at (a) $h=0.05 J, \epsilon=0.2$ and (b) $h=0.05 J, \epsilon=0.5$. The first peaks for $\epsilon=0.2$ and 0.5 lie at $\omega=0.87 \mathrm{~J}$ and $0.56 \mathrm{~J}$, respectively, which are in good agreement with the value $E_{1}(h=0.05 \mathrm{~J})=0.87 \mathrm{~J}$ and $0.54 \mathrm{~J}$, respectively, extracted from Fig. 11. Some spurious oscillations appear in (a) in spite of the damping employed in the Fourier transformation. In (b), as a result of the numerical extrapolation procedure employed to deal with the late-time regime, no spurious oscillations are visible.

for the wave functions of the excited states, we can compute the energies and spectral weights for all states contributing to the DSF.

As it works directly in the momentum-energy plane, and does not suffer from finite-size effects, this method has access to the dynamical structure factor with perfect resolution. The only source of error is the variational nature of the approach, but the approximation can be systematically improved by growing the bond dimension of the MPS ground state. As the ansatz effectively exploits the correlations in the ground state to build an excitation, it can treat generic strongly correlated spin chains with isolated branches in the spectrum to very high precision. An assessment of the accuracy of the variational wave function for a given excited state is provided by the variance $\left\langle H^{2}\right\rangle-\langle H\rangle^{2}$ of its energy, which can be evaluated exactly [53].

At small values of the magnetic field $h$, we have a large number of stable bound states that live on a strongly correlated background. Whereas time-domain approaches are necessarily 


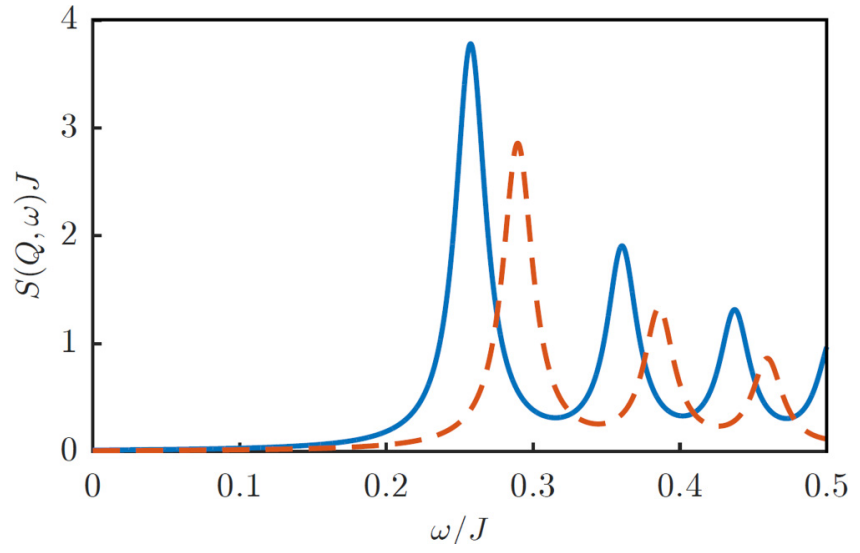

FIG. 13. Transverse (solid blue line) and longitudinal (dashed red line) structure factors for $J=7 \mathrm{meV}, \epsilon=0.56$, and $h=0.00643 J$. A Lorentzian broadening with width $\eta=J / 80$ has been introduced to make the $\delta$-function peaks visible.

limited in resolving the different modes, the quasiparticle ansatz is ideally suited for capturing all stable bound states with perfect resolution. Targeting the unstable bound states in the continuous bands would require a multiparticle ansatz [52], but this has not proven to be necessary here. In order to compare with the experimental data, we have determined the dynamical structure factor by this method for several values of the anisotropy $\epsilon$ and the staggered magnetic field $h$. The best agreement with the experimental data is found for $\epsilon=0.56$ and $h=0.00643 \mathrm{~J}$, and the corresponding structure factors are shown in Fig. 13. The detailed comparison between MPS results and the experimental data are given below in the next section (Sec. V).

\section{Comparison with strong-coupling expansion and DMRG results}

It is useful to compare the results obtained by our different methods. We first consider a fairly strong anisotropy $\epsilon=0.1$ and weak field $h=0.025 \mathrm{~J}$. Results for the quasiparticle ansatz (solid line) and the strong-coupling approach (dashed line) are shown in Fig. 14. The agreement is seen to be good and any

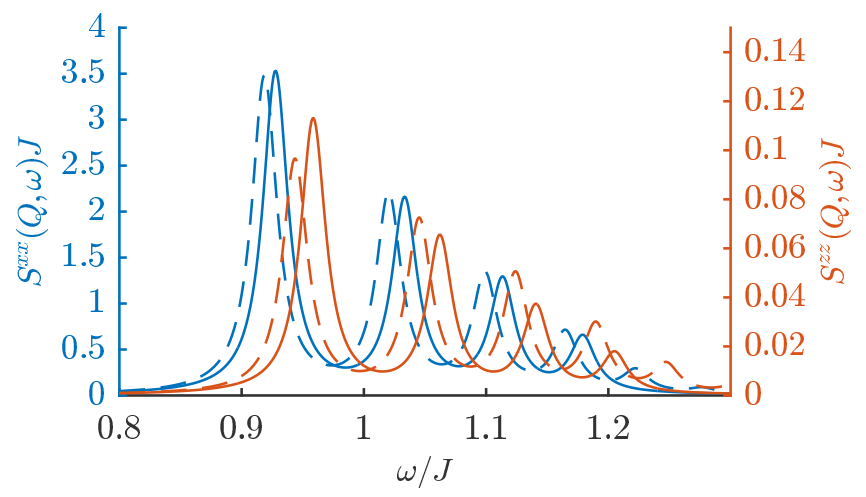

FIG. 14. Transverse (blue lines) and longitudinal (red lines) structure factors for $J=7 \mathrm{meV}, \epsilon=0.1$, and $h=0.025 J$. The results of the MPS quasiparticle ansatz (solid lines) and the strongcoupling approach (dashed lines) are in good agreement.

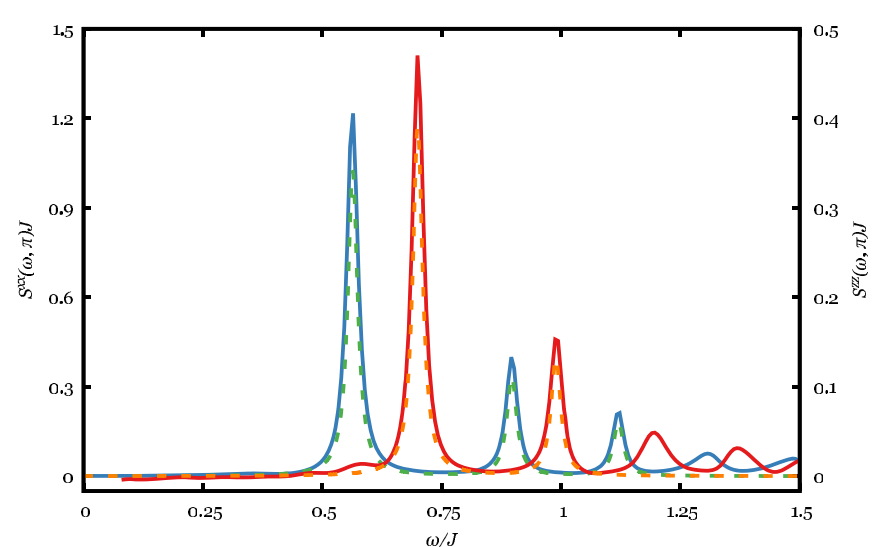

FIG. 15. Transverse (blues lines, left axis) and longitudinal (red lines, right axis) structure factors for $J=7 \mathrm{meV}, \epsilon=0.5$, and $h=$ $0.05 J$ calculated by DMRG (solid lines) and tangent-space MPS methods (dashed lines). For the chosen parameter set the agreement is seen to be excellent.

discrepancies can be attributed to the leading $O\left(\epsilon^{2}\right)$ corrections to the strong-coupling result.

We have also compared the results of the quasiparticle ansatz to DMRG for $\epsilon=0.5$; cf. Fig. 15. The two methods are seen to agree very well. The remaining differences arise from the fact that the tangent-space MPS approach has been restricted to the calculation of three lowest transversal modes and two lowest longitudinal modes. In principle, the MPS approach could be extended to include the higher energy bound modes as well, but this was not necessary for the present purposes.

\section{Temperature effects}

So far our theoretical analysis has been restricted to zero temperature. In order to access the $T>0$ regime we now combine the tangent-space MPS method with a low-temperature linked cluster expansion of the dynamical susceptibility [28,29,54,55]. The basic idea is to treat the low-temperature regime as a gas of bound states that scatter purely elastically. This is expected to be a good approximation as long as the temperature is small compared to the minimal gap $\Delta_{\text {min }}$ of the lowest energy bound state, i.e.,

$$
\exp \left(-\frac{\Delta_{\min }}{k_{B} T}\right) \ll 1 .
$$

The main temperature effects are a broadening of the $T=0$ coherent single-particle peaks and the emergence of additional peaks in the dynamical structure factor, which correspond to transitions between thermally populated single-particle excitations. The first effect requires an analysis of matrix elements between single-particle and two-particle excitations. This is a nontrivial task beyond the scope of the present work. At sufficiently low frequencies $\omega<\Delta_{\text {min }}$ the second effect is easier to capture. Let us denote the single-particle excitations of the $a^{\text {th }}$ bound state with momentum $p$ by

$$
|p\rangle_{a}, \quad p \in[0, \pi]
$$


and the corresponding dispersion relations by $\epsilon_{a}(p)$. Then the leading contributions to the dynamical structure factor at low temperatures and frequencies are

$$
\begin{aligned}
\left.S^{\alpha \alpha}(\omega, Q)\right|_{0<\omega, T<\Delta_{\min }} & \approx \frac{1}{1-e^{-\omega / k_{B} T}} \sum_{a, b} \int_{0}^{\pi} \frac{d p}{\pi}\left[e^{-\epsilon_{a}(p) / k_{B} T}-e^{-\epsilon_{b}(p+Q) / k_{B} T}\right] \delta\left(\omega+\epsilon_{a}(p)-\epsilon_{b}(p+Q)\right) \mathcal{M}_{a b}(p, Q), \\
\mathcal{M}_{a b}(p, Q) & =\left|{ }_{a}\left\langle p\left|S_{0}^{\alpha}\right| p+Q\right\rangle_{b}\right|^{2}+\left.\left.\right|_{a}\left\langle p\left|S_{1}^{\alpha}\right| p+Q\right\rangle_{b}\right|^{2}+2 \operatorname{Re}\left[e^{-i Q}{ }_{a}\left\langle p\left|S_{1}^{\alpha}\right| p+q\right\rangle_{b b}\left\langle p+Q\left|S_{0}^{\alpha}\right| p\right\rangle_{a}\right] .
\end{aligned}
$$

In Fig. 16 we show the contributions (44) due to transitions between thermally excited bound modes for the experimentally relevant parameter set $\epsilon=0.56, J=7 \mathrm{meV}, h=0.00643 J$, and $T=4 \mathrm{~K}$. Transitions occurring at very low frequencies have not been taken into account, because the low-energy regime is dominated by the broadened Bragg peak. For comparison the longitudinal (red dashed line) and transverse (solid blue line) components of the dynamical structure factor at $T=0$ are shown as well. A finite-temperature resonance in the longitudinal structure factor at a frequency $\omega \approx 0.1 \mathrm{~J}$ is clearly visible, while contributions to the transverse structure factor are very small.

\section{THEORY VS EXPERIMENT}

We are now in a position to compare theoretical and experimental results. The first task is to determine appropriate parameters for applying the effective 1D model [Eq. (13)] to $\mathrm{SrCo}_{2} \mathrm{~V}_{2} \mathrm{O}_{8}$. Estimates for the exchange $J$ and anisotropy $\epsilon$ were obtained in Sec. III A by comparing the data collected for $T>T_{N}$ to the zero-temperature transverse dynamical structure factor for [Eq. (13)] with $h=0$. Such a comparison is appropriate because $T \ll J / K_{B}$ and gives values of $J \approx 7.0$ $\mathrm{meV}$ and $\epsilon=0.56$. The remaining parameter is the strength $h$ of the effective staggered field. As this arises from a mean-field decoupling of the interchain interactions, it is temperature dependent. As shown in Sec. IV, $h$ can be fixed by computing the energies of the first few bound states and comparing them to the

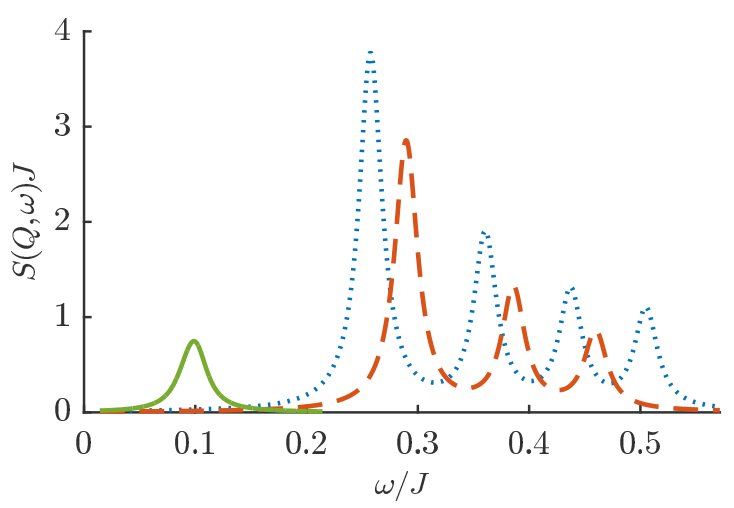

FIG. 16. Dynamical structure factor calculated with tangentspace MPS methods for $\epsilon=0.56, J=7 \mathrm{meV}, h=0.00643 J$. The temperature induced resonance at $T=4 \mathrm{~K}$, determined by Eq. (44), is shown by the green solid line. The curve is plotted together with the $T=0 \mathrm{~K}$ MPS results for the longitudinal (dashed) and transverse (dotted) bound modes to compare its intensity with that of the bound modes. For all the modes a Lorentzian broadening with width $\eta=J / 80$ has been introduced to make the $\delta$-function peaks visible. measured peak positions. One caveat is that the gap of the lowest bound state is not necessarily well described by the purely 1D model [Eq. (13)]. Indeed, in simple quasi-1D systems of weakly coupled chains corrections to the simple mean-field approximation due to the interchain couplings can be taken into account by a random-phase approximation [37], which gives the following expression for the dynamical susceptibility:

$$
\chi_{3 \mathrm{D}}(\omega, \mathbf{q})=\frac{1}{\chi_{1 \mathrm{D}}^{-1}(\omega, q)-J_{\mathrm{int}}(\mathbf{q})} .
$$

Here $J_{\text {int }}(\mathbf{q})$ is the Fourier transform of the interchain coupling, and we have assumed that we are dealing with a system of equivalent chains. It is clear from [Eq. (45)] that at a given wave vector the singularities of $\chi_{3 \mathrm{D}}(\omega, \mathbf{q})$ are shifted in energy by a constant compared to those of $\chi_{1 \mathrm{D}}(\omega, q)$. The situation in $\mathrm{SrCo}_{2} \mathrm{~V}_{2} \mathrm{O}_{8}$ is much more complicated, because there are several counter-rotating screw chains per unit cell. A refined analysis of the interchain coupling by a generalization of [Eq. (45)] is possible [56] but beyond the scope of this work.

Keeping this discussion in mind, we first try to obtain an optimal description of the energy splittings between the observed coherent modes by a pure one-dimensional model. At temperature $T=1.5 \mathrm{~K}$ we can reproduce the energy differences of the first few peaks with a value of $h \approx 0.00643 J$. The resulting comparison between the transverse modes calculated by this mean-field model using the tangent-space MPS method [Eq. (13)] and the experimental data at $Q=$ $(0,0,2)$ is shown in Fig. 17. We see that the mean-field model reproduces the experimental results very well up to an overall shift of about $0.122 \mathrm{meV}$ in energy. Since the gap is very

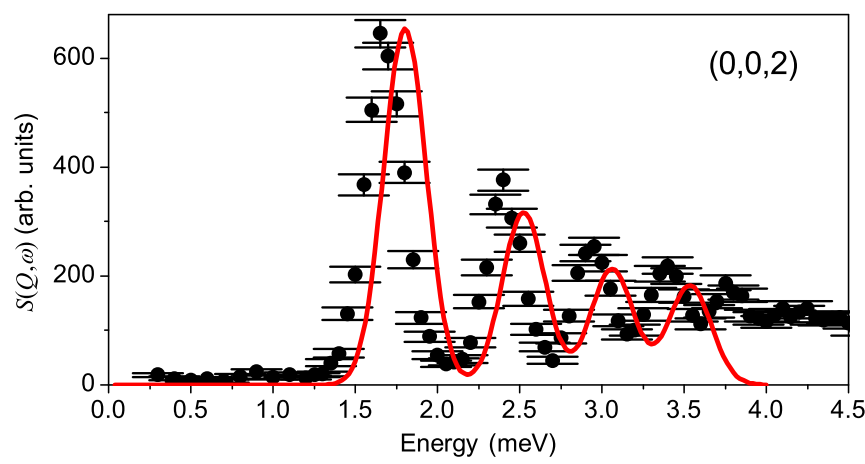

FIG. 17. Transverse dynamical structure factor calculated with tangent-space MPS methods for $\epsilon=0.56, J=7 \mathrm{meV}, h=$ $0.00643 J$ (solid line) compared to experimental data for $T=1.5 \mathrm{~K}$ and wave vector transfer $(0,0,2)$. An artificial Gaussian broadening with width $0.26 \mathrm{meV}$ was introduced to mimic the experimental resolution. The MPS results were rescaled in order to match the amplitude of the first peak. The MPS computation was restricted to determining the first four bound modes only. 


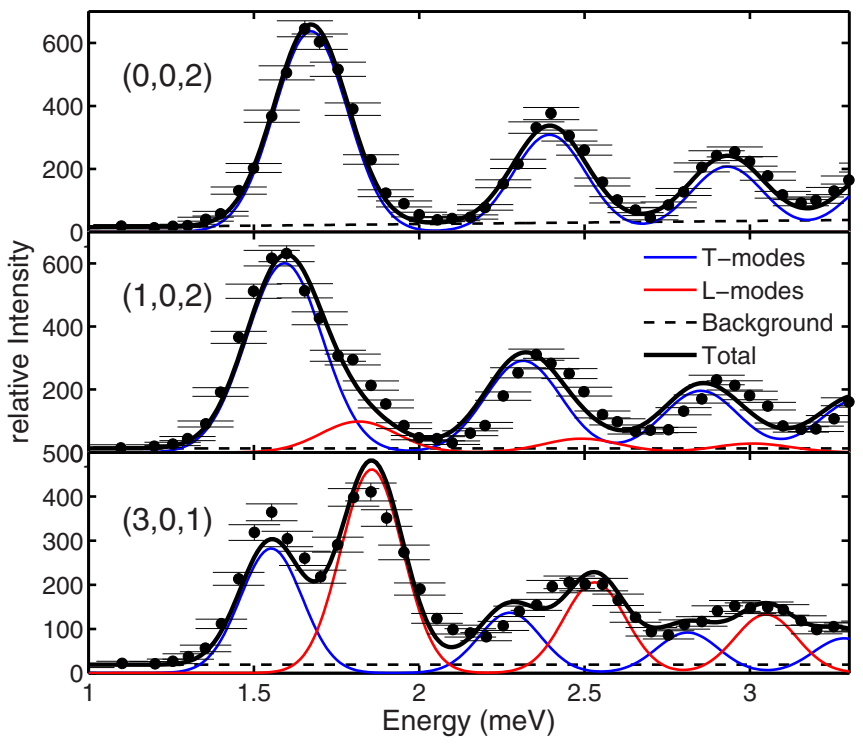

FIG. 18. The transverse (blue line) and longitudinal (red line) dynamical structure factors calculated by the tangent-space MPS method for $\epsilon=0.56, J=7 \mathrm{meV}, h=0.00643 J$ compared to the experimental data (filled circles) for $T=1.5 \mathrm{~K}$ measured at wave vector transfers (a) $(0,0,2),(b)(1,0,2)$, and (c) $(3,0,1)$. At each wave vector the intensities of the two structure factors are weighted by their respective polarization factors and by the square of their $g$ factors [57] giving a weighting ratio of longitudinal to transverse modes of $0,0.216,2.16$ for $(0,0,2),(1,0,2)$, and $(3,0,1)$, respectively; an overall scale factor is also included to match the data. To account for the effects of interchain coupling a wave-vector-dependent energy shift is introduced with values $-0.13,-0.21,-0.25 \mathrm{meV}$ for the three wave vectors, respectively. Finally the theoretical peaks are convolved by Gaussians of widths $0.26,0.27$, and $0.22 \mathrm{meV}$ to model the experimental resolution. The two structure factors are summed together along with a linear background (dashed black line) to give the expected total scattering (solid black line).

sensitive to corrections to the mean-field model, as can be seen from the RPA expression [Eq. (45)], such a shift is not surprising. Furthermore in the experimental data the interchain couplings give rise to a dispersion of the gap with a comparable bandwidth of $\sim 0.15 \mathrm{meV}$ (see Fig. 5).

The dynamical structure factor calculated in the mean-field model by the tangent-space MPS method was compared to the data at several reciprocal lattice points as shown in Fig. 18. Both the transverse and longitudinal structure factors are plotted and the effect of interchain coupling is taken into account by introducing a wave-vector-dependent energy shift. At each wave vector the intensities of the two structure factors are weighted by their respective polarization factors due to the component of their magnetization perpendicular to the wave vector transfer (see Sec. III B) as well as by the square of their $g$ factors [57]. An overall scaling factor for each wave vector is also introduced to match the theoretical intensity to the data and the theoretical peaks are convolved by a Gaussian to model the experimental resolution. The solid black line gives the sum of the two structure factors as well as a linear background and represents the expected neutron scattering intensities. Considering the highly complex

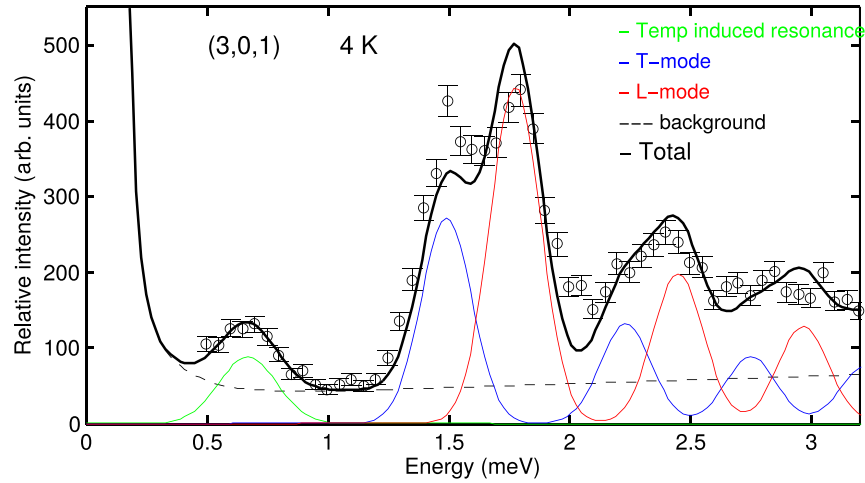

FIG. 19. A comparison of the dynamical structure factors for the transverse (blue line) and longitudinal (red line) and the temperatureinduced resonance (green line) calculated by the tangent-space MPS method (Fig. 16) with the experimental data (open circles) at $T=4$ $\mathrm{K}$ and measured at wave vector transfers $(3,0,1)$. The temperatureinduced resonance (green line) given by (44) has been determined at temperature $T=4 \mathrm{~K}$, whereas the dynamical structure factors for the $\mathrm{T}$ mode and $\mathrm{L}$ mode are calculated at $T=0 \mathrm{~K}$. The relative intensities for the $\mathrm{T}$ mode, $\mathrm{L}$ mode, and temperature-induced resonance peak are taken to be 1, 2.16 (same as Fig. 18), and 0.325, respectively. An overall scale factor is also included to match the data. For L mode and $\mathrm{T}$ mode, energy shifts of -0.31 and $-0.25 \mathrm{meV}$, respectively, are introduced to account for the effects of interchain coupling. Finally the theoretical peaks are convolved by Gaussians of widths 0.24 for the temperature-induced peak and $0.22 \mathrm{meV}$ for the $\mathrm{L}$ and $\mathrm{T}$ modes to model the experimental resolution. All the structure factors are summed together along with a background (dashed black line) to give the expected total scattering (solid black line). The background was calculated by a combination of two functions: a linear and an exponential decay for which the coefficients are derived from the fitting of the $4.75 \mathrm{~K}$ experimental data for wave vector transfer $(0,0,2)$ [Fig. 7(a)].

counter-rotating screw chain structure and the many possible interchain interactions that are neglected in this calculation, the agreement between experiment and theory is remarkably good.

The finite-temperature results of Sec. IV D 2 are also in good agreement with the experimental observations (Fig. 19). We saw that at a temperature of $4 \mathrm{~K}$ our one-dimensional model displays a finite-temperature resonance in the longitudinal structure factor at an energy of about $0.7 \mathrm{meV}$. This is in good agreement with the experimental observation of a resonance in the longitudinal structure factor at $\omega \approx 0.65 \mathrm{meV}$ [Fig. 7(b)].

\section{DISCUSSION}

We have presented results of inelastic neutron scattering experiments on the quasi-one-dimensional spin- $1 / 2$ Heisenberg magnet $\mathrm{SrCo}_{2} \mathrm{~V}_{2} \mathrm{O}_{8}$. Above the Néel temperature $T_{N} \approx$ $5.2 \mathrm{~K}$, the neutron scattering cross section is dominated by a scattering continuum that is well described by a spin- $1 / 2$ Heisenberg XXZ chain with antiferromagnetic exchange $J \approx$ $7.0 \pm 0.2 \mathrm{meV}$ and anisotropy parameter $\epsilon \approx 0.56 \pm 0.02$. The scattering continuum is formed by fractionalized $S^{z}=\frac{1}{2}$ spinon excitations. At temperatures below $T_{N}$ the structure factor exhibits two sequences of resolution-limited dispersing peaks that are associated with fluctuations along ( $\mathrm{L}$ modes) 
and perpendicular (T modes) to the ordered magnetic moment, respectively.

The origin of these coherent modes can be understood by a one-dimensional model [Eq. (13)], in which a (temperaturedependent) staggered magnetic field is generated in the ordered phase through a mean-field decoupling of the interchain interactions. The model can be studied analytically for both strong and weak exchange anisotropies and in both limits the effect of the staggered field is to confine the spinon excitations into two sequences of bound states. At intermediate values of the exchange anisotropy we have used DMRG and MPS methods to obtain quantitative results for the dynamical structure factor. It turns out that the experimentally relevant parameter regime cannot be reached even by state-of-the-art DMRG methods. Due to entanglement growth, the time scale by which dynamical correlation functions that can be computed by DMRG is restricted, which in turn imposes limitations on the achievable energy resolution. We therefore have employed a recently developed tangent-space MPS method, which is based on constructing MPS representations for excited states. Application of this method allows the computation of the dynamical structure factor, which is found to be in good agreement with experiment.

Our work establishes $\mathrm{SrCo}_{2} \mathrm{~V}_{2} \mathrm{O}_{8}$ as a beautiful paradigm for spinon confinement in a quasi-one-dimensional quantum magnet. There are a number of interesting questions that deserve further investigation. On the theoretical side a more involved investigation of the dynamical structure factor at finite temperatures would improve our understanding of the thermally induced peaks observed in the data both below $T_{N}$ (transitions between bound modes) and above $T_{N}$ (the villain mode). On the experimental side, the precise form of the interchain interactions needs to be clarified by extensive measurements of the bound mode dispersion relations perpendicular to the chain direction at lowest temperatures. We have seen that it is necessary to account for these interactions beyond a simple mean-field decoupling in order to describe the data. As the crystal structure is rather complex this goes beyond the scope of the present work. Finally, it would be interesting to analyze the effects of an applied uniform magnetic field. Terahertz spectroscopy measurements reveal the emergence of novel excitations as a function of both transverse and longitudinal magnetic field $[57,58]$ which could be investigated using a combination of neutron scattering and the theoretical methods described here. We hope to return to these questions in future work.

\section{ACKNOWLEDGMENTS}

We acknowledge the Helmholtz Gemeinschaft for funding via the Helmholtz Virtual Institute (Project No. HVI-521). This work was supported by the EPSRC under Grant No. EP/N01930X/1 (F.H.L.E.), and the ExQM graduate school and the Nanosystems Initiative Munich (C.H.). L.V. acknowledges financial support from FWO Flanders.
[1] S. Sachdev, Quantum Phase Transitions (Cambridge University Press, Cambridge, 2011).

[2] B. Lake, D. A. Tennant, C. D. Frost, and S. E. Nagler, Quantum criticality and universal scaling of a quantum antiferromagnet, Nat. Mater. 4, 329 (2005).

[3] M. Hälg, D. Hüvonen, T. Guidi, D. L. Quintero-Castro, M. Boehm, L. P. Regnault, M. Hagiwara, and A. Zheludev, Finitetemperature scaling of spin correlations in an experimental realization of the one-dimensional Ising quantum critical point, Phys. Rev. B 92, 014412 (2015).

[4] T. Giamarchi, C. Regg, and O. Tchernyshyov, Bose-Einstein condensation in magnetic insulators, Nat. Phys. 4, 198 (2008).

[5] I. Affleck, Bose condensation in quasi-one-dimensional antiferromagnets in strong fields, Phys. Rev. B 43, 3215 (1991).

[6] B. Thielemann, Ch. Rüegg, H. M. Ronnow, A. M. Läuchli, J.-S. Caux, B. Normand, D. Biner, K. W. Krämer, H.-U. Güdel, J. Stahn, K. Habicht, K. Kiefer, M. Boehm, D. F. McMorrow, and J. Mesot, Direct Observation of Magnon Fractionalization in the Quantum Spin Ladder, Phys. Rev. Lett. 102, 107204 (2009).

[7] I. Affleck, Theory of Haldane-gap antiferromagnets in applied fields, Phys. Rev. B 41, 6697 (1990).

[8] A. M. Tsvelik, Field-theory treatment of the Heisenberg spin-1 chain, Phys. Rev. B 42, 10499 (1990).

[9] L. D. Faddeev and L. A. Takhtajan, What is the spin of a spin wave? Phys. Lett. A 85, 375 (1981).

[10] L. D. Faddeev and L. Takhtajan, J. Sov. Math. 24, 241 (1984).

[11] D. A. Tennant, R. A. Cowley, S. E. Nagler, and A. M. Tsvelik, Measurement of the spin-excitation continuum in onedimensional $\mathrm{KCuF}_{3}$ using neutron scattering, Phys. Rev. B 52, 13368 (1995).
[12] B. Lake, D. A. Tennant, and S. E. Nagler, Novel Longitudinal Mode in the Coupled Quantum Chain Compound $\mathrm{KCuF}_{3}$, Phys. Rev. Lett. 85, 832 (2000).

[13] B. Lake, D. A. Tennant, and S. E. Nagler, Longitudinal magnetic dynamics and dimensional crossover in the quasi-onedimensional spin-12 Heisenberg antiferromagnet $\mathrm{KCuF}_{3}$, Phys. Rev. B 71, 134412 (2005).

[14] B. M. McCoy and T. T. Wu, Phys. Rev. D 18, 1259 (1978).

[15] B. M. McCoy and T. T. Wu, Two-dimensional Ising field theory in a magnetic field: Breakup of the cut in the two-point function, Phys. Rev. B 18, 4886 (1978).

[16] R. Coldea, D. A. Tennant, E. M. Wheeler, E. Wawrzynska, D. Prabhakaran, M. Telling, K. Habicht, P. Smeibidl, and K. Kiefer, Quantum criticality in an Ising chain: Experimental evidence for emergent E8 symmetry, Science 327, 177 (2010).

[17] B. Lake, A. M. Tsvelik, S. Notbohm, D. A. Tennant, T. G. Perring, M. Reehuis, C. Sekar, G. Krabbes, and B. Büchner, Confinement of fractional quantum number particles in a condensed-matter system, Nat. Phys. 6, 50 (2010).

[18] B. Grenier, S. Petit, V. Simonet, E. Canévet, L.-P. Regnault, S. Raymond, B. Canals, C. Berthier, and P. Lejay, Longitudinal and Transverse Zeeman Ladders in the Ising-Like Chain Antiferromagnet $\mathrm{BaCo}_{2} \mathrm{~V}_{2} \mathrm{O}_{8}$, Phys. Rev. Lett. 114, 017201 (2015).

[19] A. K. Bera, B. Lake, W.-D. Stein, and S. Zander, Magnetic correlations of the quasi-one-dimensional half-integer spinchain antiferromagnets $\mathrm{SrM}_{2} \mathrm{~V}_{2} \mathrm{O}_{8}(\mathrm{M}=\mathrm{Co}, \mathrm{Mn})$, Phys. Rev. B 89, 094402 (2014).

[20] Z. He, T. Taniyama, and M. Itoh, Antiferromagneticparamagnetic transitions in longitudinal and transverse magnetic 
fields in a $\mathrm{SrCo}_{2} \mathrm{~V}_{2} \mathrm{O}_{8}$ crystal, Phys. Rev. B 73, 212406 (2006).

[21] A. K. Bera, B. Lake, A. T. M. N. Islam, O. Janson, H. Rosner, A. Schneidewind, J. T. Park, E. Wheeler, and S. Zander, Consequences of critical interchain couplings and anisotropy on a Haldane chain, Phys. Rev. B 91, 144414 (2015).

[22] A. Abragam and B. Bleaney, Electron Paramagnetic Resonance of Transition Ions (Clarendon, Oxford, 1970).

[23] J. C. Bonner and M. E. Fisher, Linear magnetic chains with anisotropic coupling, Phys. Rev. 135, A640 (1964).

[24] J.-S. Caux, J. Mossel, and I. Përez-Castillo, The two-spinon transverse structure factor of the gapped Heisenberg antiferromagnetic chain, J. Stat. Mech. (2008) P08006.

[25] J. Villain, Propagative spin relaxation in the Ising-like antiferromagnetic linear chain, Physica B+C 79, 1 (1975).

[26] S. E. Nagler, W. J. L. Buyers, R. L. Armstrong, and B. Briat, Propagating Domain Walls in $\mathrm{CsCoBr}_{3}$, Phys. Rev. Lett. 49, 590 (1982).

[27] S. E. Nagler, W. J. L. Buyers, R. L. Armstrong, and B. Briat, Solitons in the one-dimensional antiferromagnet $\mathrm{CsCoBr}_{3}$, Phys. Rev. B 28, 3873 (1983).

[28] W. D. Goetze, U. Karahasanovic, and F. H. L. Essler, Lowtemperature dynamical structure factor of the two-leg spin-1/2 Heisenberg ladder, Phys. Rev. B 82, 104417 (2010).

[29] A. J. A. James, F. H. L. Essler, and R. M. Konik, Finitetemperature dynamical structure factor of alternating Heisenberg chains, Phys. Rev. B 78, 094411 (2008).

[30] A. J. A. James, W. D. Goetze, and F. H. L. Essler, Finitetemperature dynamical structure factor of the Heisenberg-Ising chain, Phys. Rev. B 79, 214408 (2009).

[31] Z. Wang, M. Schmidt, A. K. Bera, A. T. M. N. Islam, B. Lake, A. Loidl, and J. Deisenhofer, Spinon confinement in the onedimensional Ising-like antiferromagnet $\mathrm{SrCo}_{2} \mathrm{~V}_{2} \mathrm{O}_{8}$, Phys. Rev. B 91, 140404 (2015).

[32] H. Shiba, Quantization of magnetic excitation continuum due to interchain coupling in nearly one-dimensional Ising-like antiferromagnets, Prog. Theor. Phys. 64, 466 (1980).

[33] P. Fonseca and A. B. Zamolodchikov, Ising Spectroscopy I: Mesons at $T<T_{c}$, arXiv:hep-th/0612304.

[34] M. J. Bhaseen and A. M. Tsvelik, in From Fields to Strings: Circumnavigating Theoretical Physics, edited by M. Shifman, A. Vainshtein, and J. Wheater (World Scientific, Singapore, 2005).

[35] S. B. Rutkevich, On the weak confinement of kinks in the one-dimensional quantum ferromagnet $\mathrm{CoNb}_{2} \mathrm{O}_{6}$, J. Stat. Mech. (2010) P07015.

[36] L. D. Landau and E. M. Lifshitz, Quantum Mechanics, 3rd ed. (Butterworth-Heinemann, Oxford, 1999).

[37] H. J. Schulz, Dynamics of Coupled Quantum Spin Chains, Phys. Rev. Lett. 77, 2790 (1996).

[38] F. H. L. Essler, A. M. Tsvelik, and G. Delfino, Quasi-onedimensional spin-1/2 Heisenberg magnets in their ordered phase: Correlation functions, Phys. Rev. B 56, 11001 (1997).

[39] A. W. Sandvik, Multichain Mean-Field Theory of Quasi-OneDimensional Quantum Spin Systems, Phys. Rev. Lett. 83, 3069 (1999).

[40] F. H. L. Essler and R. M. Konik, in From Fields to Strings: Circumnavigating Theoretical Physics, edited by M. Shifman, A. Vainshtein, and J. Wheater (World Scientific, Singapore, 2005).
[41] N. Ishimura and H. Shiba, Dynamical correlation functions of one-dimensional anisotropic Heisenberg model with spin-1/2. I: Ising-like antiferromagnets, Prog. Theor. Phys. 63, 743 (1980).

[42] A. O. Gogolin, A. A. Nersesyan, and A. M. Tsvelik, Bosonization in Strongly Correlated Systems (Cambridge University Press, Cambridge, 1999).

[43] T. Giamarchi, Quantum Physics in One Dimension (Oxford University Press, New York, 2004).

[44] I. Affleck, in Dynamical Properties of Unconventional Magnetic Systems, NATO ASI Series E, edited by A. Skjeltorp and D. Sherrington (Kluwer Academic, The Netherlands, 1998), Vol. 349.

[45] G. Delfino and G. Mussardo, Non-integrable aspects of the multi-frequency sine-Gordon model, Nucl. Phys. B 516, 675 (1998); G. Mussardo, V. Riva, and G. Sotkov, Semiclassical particle spectrum of double sine-Gordon model, ibid. $\mathbf{6 8 7}, 189$ (2004).

[46] S. White, Density Matrix Formulation for Quantum Renormalization Groups, Phys. Rev. Lett. 69, 2863 (1992).

[47] U. Schollwöck, The density-matrix renormalization group in the age of matrix product, Ann. Phys. 326, 96 (2011).

[48] C. Hubig, I. P. McCulloch, U. Schollwöck, and F. A. Wolf, Strictly single-site DMRG algorithm with subspace expansion, Phys. Rev. B 91, 155115 (2015).

[49] P. E. Dargel, A. Wöllert, A. Honecker, I. P. McCulloch, U. Schollwöck, and T. Pruschke, Lanczos algorithm with matrix product states for dynamical correlation functions, Phys. Rev. B 85, 205119 (2012).

[50] J. Haegeman, T. J. Osborne, and F. Verstraete, Post-matrix product state methods: To tangent space and beyond, Phys. Rev. B 88, 075133 (2013).

[51] J. Haegeman, B. Pirvu, D. J. Weir, J. I. Cirac, T. J. Osborne, H. Verschelde, and F. Verstraete, Variational matrix product ansatz for dispersion relations, Phys. Rev. B 85, 100408(R) (2012).

[52] L. Vanderstraeten, J. Haegeman, F. Verstraete, and D. Poilblanc, Quasiparticle interactions in frustrated Heisenberg chains, Phys. Rev. B 93, 235108 (2016).

[53] L. Vanderstraeten, F. Verstraete, and J. Haegeman, Scattering particles in quantum spin chains, Phys. Rev. B 92, 125136 (2015).

[54] F. H. L. Essler and R. M. Konik, Finite-temperature line shapes in gapped quantum spin chains, Phys. Rev. B 78, 100403 (2008).

[55] F. H. L. Essler and R. M. Konik, Finite-temperature dynamical correlations in massive integrable quantum field theories, J. Stat. Mech. (2009) P09018.

[56] A. Zheludev, T. Masuda, I. Tsukada, Y. Uchiyama, K. Uchinokura, P. Böni, and S.-H. Lee, Magnetic excitations in coupled Haldane spin chains near the quantum critical point, Phys. Rev. B 62, 8921 (2000).

[57] Z. Wang, J. Wu, S. Xu, W. Yang, C. Wu, A. K. Bera, A. T. M. N. Islam, B. Lake, D. Kamenskyi, P. Gogoi, H. Engelkamp, N. Wang, J. Deisenhofer, and A. Loidl, From confined spinons to emergent fermions: Observation of elementary magnetic excitations in a transverse-field Ising chain, Phys. Rev. B 94, 125130 (2016).

[58] Z. Wang, J. Wu, W. Yang, A. K. Bera, D. Kamenskyi, A. T. M. N. Islam, S. Xu, J. M. Law, B. Lake, C. Wu, and A. Loidl, Experimental observation of Bethe strings, arXiv:1706.04181. 